\title{
Eye in the process: Formation of "triple cation" perovskite thin films rationalized by in-situ optical monitoring
}

\section{Aboma Merdasa}

Helmholtz-Zentrum Berlin

Carolin Rehermann

Helmholtz-Zentrum Berlin

Katrin Hirselandt

Helmholtz-Zentrum Berlin

Jinzhao Li

Helmholtz-Zentrum Berlin

\section{Oliver Maus}

Helmholtz-Zentrum Berlin

\section{Florian Mathies}

Helmholtz-Zentrum Berlin

Thomas Unold

Helmholtz-Zentrum Berlin

Janardan Dagar

Helmholtz-Zentrum Berlin

\section{Rahim Munir}

Helmholtz-Zentrum Berlin

Eva Unger ( $\sim$ eva.unger@helmholtz-berlin.de)

Helmholtz-Zentrum Berlin https://orcid.org/0000-0002-3343-867X

\section{Method Article}

Keywords: In-situ optical monitoring, metal-halide perovskites, formation, spin-coating, anti-solvent drip

Posted Date: November 4th, 2020

DOI: https://doi.org/10.21203/rs.3.rs-102041/v1

License: (c) (1) This work is licensed under a Creative Commons Attribution 4.0 International License.

Read Full License 


\title{
Eye in the process: Formation of "triple cation" perovskite thin films rationalized by in-situ optical monitoring
}

\author{
Aboma Merdasa ${ }^{* a, x}$, Carolin Rehermann ${ }^{a}$, Katrin Hirselandt ${ }^{a}$, Jinzhao Li $i^{a}$, Oliver Maus $^{a}$, Florian Mathies ${ }^{a}$, Thomas Unold ${ }^{b}$, \\ Janardan Dagara, Rahim Munir ${ }^{a, x}$, Eva Unger ${ }^{* a, c}$
}

\begin{abstract}
Record performance Metal-Halide Perovskite (MHP) based solar cells have been achieved by incremental optimization of deposition procedures based on spin-coating. We here provide unprecedented insight into the formation process of MHP thin films of the "triple cation" ( $\mathrm{Cs}, \mathrm{MA}, \mathrm{FA}) \mathrm{Pb}(\mathrm{Br}, \mathrm{I}) 3$ perovskite from multi-modal in-situ optical process monitoring during spin-coating and annealing. This report details small-footprint fiber-optics based optical spectroscopy setup that enables monitoring of thin-film formation processes by UV-Vis reflectance and photoluminescence spectroscopy with a sub-second time resolution. Complementary information can be obtained from optical features during different stages of film formation: 1) During the first, flow regime dominated, stage of spin-coating, the wet-film thinning can be analyzed from UV-Vis interference, 2) the onset of bulk perovskite formation is clearly observed from the evolution of the semiconductor absorption edge, and 3) Photoluminescence (PL) measurements provide complementary information on nucleation and growth processes. We here provide a comprehensive picture that rationalizes the conditions to obtain a high quality "triple cation" perovskite thin-film during spin-coating and subsequent annealing.
\end{abstract}

\section{Introduction}

The recent advent of metal halide perovskite (MHP) semiconductors represents a compelling case of high performance obtained in solution-processed solar cells, sensors, and LEDs. ${ }^{1-3}$ Perovskite solar cells (PSCs) are the best solution-processed solar cells to date rivaling established solar cell technologies. ${ }^{4}$ The highest efficiency devices are most often processed by spin-coating. ${ }^{5}$ To enable larger area devices, scalable deposition methods such as blade coating, spray coating, slot-die coating, and inkjet printing have been demonstrated. ${ }^{6-9}$ Translating optimized process conditions from spin-coating to scalable deposition methods is challenging as the rates of drying/solvent removal and the onset of crystallization are strongly dependent on the specific process conditions. An in-depth understanding of the thin film formation mechanism facilitates a knowledge-based adaptation of precursor solutions and process parameters when transferring deposition procedures to scalable solution-based deposition methods. This work provides an in-depth rationalization of one of the most utilized MHP-composition, the "triple cation" $(\mathrm{Cs}, \mathrm{MA}, \mathrm{FA}) \mathrm{Pb}(\mathrm{Br}, \mathrm{I})_{3}$ perovskite ${ }^{10}$, that has enabled fabrication of highly-efficient small-area perovskite solar cells by spin-coating reproducibly. ${ }^{11}$

Spin-coating procedures for the deposition of MHP thin-films have been iteratively optimized throughout the past years. Optimized deposition procedures to achieve high-quality thin films by spin-coating involve the use of a mixture of solvents and inducing crystallization during spin-coating, by the deposition of an anti-solvent. ${ }^{11-17}$ Exact fabrication procedures may, however, vary and have to be optimized from laboratory to laboratory as thin-film morphology and quality may be critically dependent on exact timing, temperature and atmosphere. ${ }^{16,18-22}$ Changes in precursor solution concentration upon solvent removal can be assumed to induce the onset of crystallization, which is critically affected by external factors such as temperature and ambient atmosphere. ${ }^{23}$ In metal-halide perovskite solutions, solvents are not just solubilizing agents but interact with the metal-halide precursors in specific manners and control the complex and colloidal chemistry. ${ }^{24-27}$ Solvents may be incorporated into crystalline solvate intermediate phases during the initial stages of processing, which alters the crystallization process and affects the morphology of resulting thin films. ${ }^{6,28-32}$ This ultimately paints a rather complicated picture when attempting to understand the formation processes, which is a prerequisite step if one is to systematically optimize perovskite recipes among the increasing number of viable constituents. ${ }^{33,34}$ "Blindly" optimizing processing conditions, such as the timing of anti-solvent dispense, consumes a vast amount of resources in terms of both time and material.

Monitoring film formation in-situ during different processing stage enables the assessment of parameters affecting the thin film formation during processing and hence guide process optimization. ${ }^{35-37}$ Valuable insight into different perovskite deposition strategies has been obtained from in-situ monitoring techniques such as grazing incidence wide-angle $\mathrm{X}$-ray scattering (GIWAXS), ${ }^{38-41}$ optical microscopy, ${ }^{6,42}$ and absorption measurements ${ }^{21,43,44}$ that provided critical insight guiding process optimization towards higher quality thin films and better device performance. These experiments are often carried out in specialized experimental setups. The motivation of the equipment developed and discussed herein was to enable experimentation under the same process conditions used to make thin-film samples and solar cells in our and many laboratories around the World.

We developed a small footprint assembly of an in-situ optical monitoring setup using standard optical parts that can easily be adapted to fit into processing equipment in our laboratory and are compatible with processing inside a nitrogen-filled glovebox. This setup was further developed from one previously reported by Suchan et al. ${ }^{36}$ Our system allows for directly monitoring UVVis and photoluminescence $(\mathrm{PL})$ via a simple reflectance probe, allowing for all optics and detection to remain outside the glovebox. 
We demonstrate the capabilities of the in-situ optical monitoring system using the widely adopted standard "triple cation" perovskite recipe for regular devices spin-cast from precursor solutions prepared as outlined in the supporting information. This is a standard process enabling perovskite solar cells $>20 \% .{ }^{11}$

From the analysis of optical interference and transflectance signatures, the critical stages during spin-coating can be identified. From the optical interference pattern, the thinning of the wet-film thickness during the flow and evaporation regime can be extracted with sub-second time-resolution. Initial rheological parameters obtained from optical measurements agree well with experimental reference values, and changes in solution viscosity during spin-coating can be captured. The evolution of the integral transflectance as a function of process time elapsed enables the identification of the onset of perovskite or intermediate phase crystallization. Monitoring the evolving PL spectra provides complementary information that can be attributed to the crystallization of a solid-state perovskite semiconductor thin films. While we here utilized the methodology foremost to rationalize an established standard process, these results provide a glimpse into how methodical in-situ optical analysis of film formation processes approach can guide the optimization solution process conditions for perovskite thin film deposition.

\section{Experimental Setup \& Methods}

A schematic of the experimental setup is shown in Figure 1a. We use a reflectance probe (Ocean Insight part QR400-7-UV-BX) to probe absorption and emission of samples during processing, which can be conveniently mounted in very close proximity of ca. 1 $\mathrm{cm}$ with respect to the surface of the sample under investigation enabling good signal to noise and fast acquisition rates. ${ }^{45}$ The strategic use of a reflectance probe eliminates the need for two separate probes where excitation and detection are decoupled. A halogen lamp (Thorlabs model SLS201LM) was used for absorption measurements and LEDs with peak emission wavelengths at $365 \mathrm{~nm}, 470 \mathrm{~nm}$, or $625 \mathrm{~nm}$ as excitation light sources for PL experiments. An Ocean Insight fiber-optic spectrometer (FLMS12200) was used as the detector. Using a custom design glovebox feed-through (VACOM), only the reflectance probe is needed inside the glovebox for experiments, while other components such as light sources and detectors can be kept outside. This minimizes potential damage of equipment due to chemical or solvent vapors and minimizes the footprint of the setup enabling easy integration into existing process equipment. The reflectance probe is mounted using standard Thorlabs optomechanics, which can be customized to fit a variety of processing equipment. With the components described here, we are able to collect spectra in the range between $340 \mathrm{~nm}-1040 \mathrm{~nm}$ with millisecond temporal resolution. Depending on the light source coupled into the reflectance probe, we measure either photoluminescence or 'absorptance in reflection mode,' meaning that incoming light will pass through the absorbing material twice. As the absorption signal will be affected by changes in sample absorptivity and scattering, we here chose to refer to the absorption signals measured as 'transflectance' to highlight the specific measurement geometry employed. Signal acquisition was carried out based on a customized LabVIEW automated acquisition routine.

The inset in Figure 1a shows the schematic of the measurement geometry, including dimensions of the internal fibers of the reflectance probe that both bring light to and from the sample and the distance of the probe from the sample surface during experimentation. The fibers have a NA of 0.22 , which means the incident light escapes the fiber at an angle of $\theta_{e}=13^{\circ}$ from the normal. However, from the reflected light, only that within a narrow angular range $\left(\theta_{d}=1-3^{\circ}\right)$ is detected, which has implications for thickness estimations, as will be discussed below.

To detect a reliable signal, it is therefore favorable if the bare substrate reflects as much of the incoming light as possible. To increase the amount of light reflected back to the detector, we evaporated a thin layer of silver onto the backside of the substrate, as previously reported by Suchan at al. ${ }^{36}$ To maximize information gained from interference patterns, we performed measurements with the precursor solution being deposited on top of the silver-coated surface of the substrate, as discussed in SI Note 4. Introducing a buffer or transport layer in-between, such as PTAA, avoids unwanted chemical reactions between the perovskite and the Ag layer.

A white reference spectrum, $I(\lambda)_{B}$, is acquired using the bare substrate before perovskite deposition. A dark reference spectrum, $I(\lambda)_{D}$, is recorded with the excitation light off. The UV-Vis 'transflectance' signal, (TF), is calculated as

$T F(\lambda)=1-\frac{I(\lambda)_{S}-I(\lambda)_{D}}{I(\lambda)_{B}-I(\lambda)_{D}}$

where $\mathrm{I}(\lambda)_{\mathrm{s}}$ is the dynamic signal measured for the sample under investigation. 


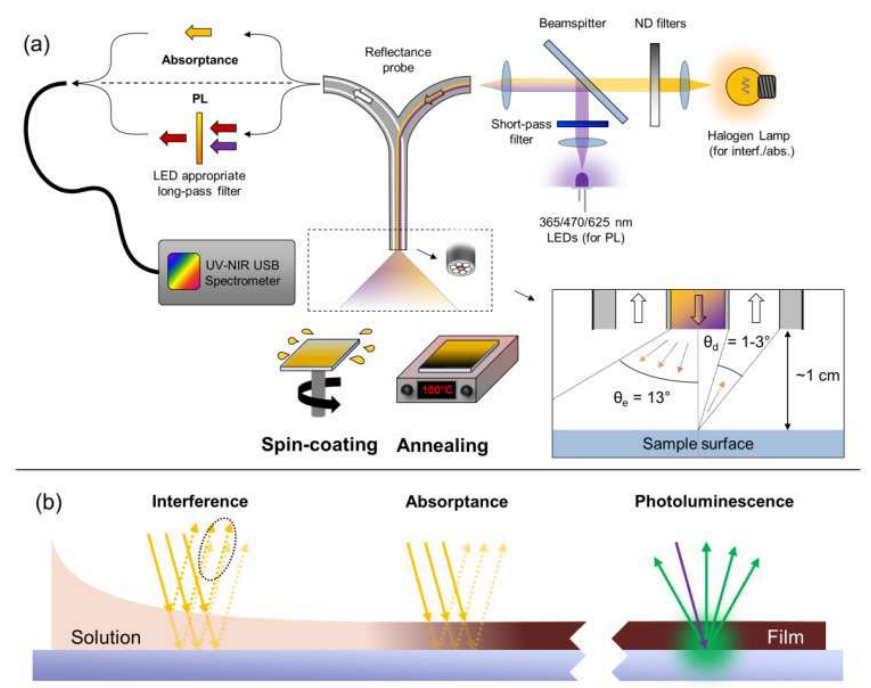

Figure 1. (a) Schematic representation of the measurement setup in which only the reflectance probe is inside the glovebox. A white light source (halogen lamp) is used for absorptance measurements a,nd one of three available LEDs (emission peak at $365 \mathrm{~nm}, 470 \mathrm{~nm}$, or $625 \mathrm{~nm}$ ) is used for photoluminescence (PL) generation. The reflectance probe can be placed directly above either the spin-coater or hot plate. Light is detected with a USB spectrometer (Ocean Insight) prior to which an LED appropriate long-pass filter is placed for PL measurements to filter out the excitation. (b) Schematic demonstrating the thinning and progression from solution to a crystallized film during spin-coating. The schematic also illustrates the three types of measurements we can employ during this process, where we can measure the interference pattern (extracting thickness), the absorptance, or in a separate measurement, the PL.

A rougher sample surface upon crystallization is expected to change light scattering dramatically and results in a characteristic baseline shift increasing towards the blue part of the spectrum. The formation of absorbing species in the visible part of the spectrum should give rise to distinctive absorption features. For the specific case investigated here, the formation of the perovskite semiconductor should give rise to a step-wise absorption onset around $1.7 \mathrm{eV}$. As will be demonstrated, analysis of changes in TF above and below the absorption onset enables us to distinguish between changes in sample reflectivity due to the formation of a crystalline species from the more specific formation of a perovskite semiconductor.

As the photoluminescence $(\mathrm{PL})$ is emitted isotropically and the detection angle is similar to the incident angle $\left(\sim 13^{\circ}\right)$, we assume a similar fraction of PL being detected during the course of the experiment. PL in the experiments demonstrated herein is generated using a $470 \mathrm{~nm}$ LED light source, but light sources with different excitation wavelengths are available and easily exchanged in our modular setup. While the area of the film that receives the excitation can be adjusted by changing the height of the reflectance probe (at the cost of a reduced intensity), we are still only collecting a very narrow angular range of the isotropic emission. This is a crucial point to keep in mind when measuring perovskites due to the widely reported sensitivity to light and its influence on the optoelectronic properties. ${ }^{46}$ Keeping the reflectance probe at a low height of $1 \mathrm{~cm}$ will illuminate a region that is roughly $0.5 \mathrm{~cm}$ in diameter. If the substrate is larger (i.e., 1 square inch), the probe can be shifted laterally so that the probe is offcentered from the rotating sample, which reduces the light exposure time and provides a spatially averaged signal ${ }^{47,48}$. This is

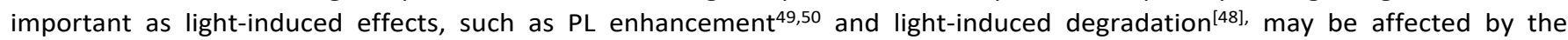
experimental results of optical in-situ monitoring experiments.

The schematic in Figure $1 \mathrm{~b}$ visualizes different stages of thin-film formation during spin-coating, where the thinning of the wet film is derived from interference and the onset of perovskite crystallization from the evolution of the bandgap in an absorption spectrum. The break in the schematic between PL and the other measurements denotes that these are measured in two separate measurements. It would be possible to measure both PL and absorptance in short alternating time intervals ${ }^{[49]}$, but this requires an increased complexity in the experimental setup. What is unique with the method presented here is that we can extract both thinning (via interference) and crystallization onset via absorption in the same measurement.

We here investigated the evolution of spectral features during deposition by spin-coating and annealing of "triple cation" perovskite precursor solutions prepared according to Saliba et al., as specified in the supporting information (SI). Resulting precursor solutions with an approximate ion precursor concentration of $1.2 \mathrm{M}\left(0.06 \mathrm{M} \mathrm{Csl}, 0.20 \mathrm{M} \mathrm{MAPbBr}_{3}\right.$ and $\left.0.98 \mathrm{M} \mathrm{FAPbl}_{3}\right)$ in $\mathrm{N}, \mathrm{N}$-dimethylformamide (DMF) and dimethylsulfoxide (DMSO) solvent in a 4:1 mixture. For experiments on lower precursor concentrations, solutions were diluted with the DMF/DMSO solvent mixture. Rheological properties of solution density, viscosity and refractive index of the precursor inks with varying concentrations were determined using a rolling ball viscometer and an Abbé refractometer (see SI for details).

\section{Results}


During spin-coating, the precursor solution is during the initial stages expected to be thinning and spreading, which we will discuss in section 3.1 based on our analysis of changes in the interference pattern. We here discuss differences between spin-coating speed as an example of the information that can be gained using our setup. As MHP semiconductors crystallize during spin-coating, the thin film morphology will be strongly affected by processing conditions. In section 3.2. we discuss the perovskite formation derived from in-situ absorption measurements as a function of the precursor solution concentration. In section 3.3. we discuss the role of an anti-solvent drip deposited during spin-coating monitored by UV-Vis absorption and in-situ photoluminescence (PL). We conclude in section 3.4. with the full picture of the triple-cation MHP formation process during spin-coating and include spectral evolution during the annealing step.

\section{1. Monitoring the Thinning Behavior of Wet Films from Interference}

In Figure 2, the effect of spin-coating speed on the thinning behavior of the film prior to crystallization is demonstrated. Figures $2 a-b$ show the evolution of the transflectance interference pattern in the representation as 2D heat maps, where time is at abscissa and wavelength is at the ordinate. We compare the evolving interference pattern during $60 \mathrm{~s}$ of spin-coating perovskite precursor solutions at $2000 \mathrm{rpm}$ (Figure 2a) and $6000 \mathrm{rpm}$ (Figure 2b). For both measurements, the number of fringes reduces with time. This is consistent with the wet film thinning during spin-coating. As expected, the rate at which the number of fringes reduces is quicker for the film spin-coated at higher rpm. This is evident from the two interference patterns extracted after the same time elapsed for the two measurements (Figures $2 a_{1}, b_{1}$ ).

By analyzing the interference patterns created from the fraction of the incident light reflected from the top surface of the drying film and substrate, the wet film thickness can be extracted. Whether the light interferes constructively or destructively will depend on the additional path length of the light reflected off the substrate, which in turn depends on the refractive index ( $n$ ) of the material, the angle of incidence $(\theta)$ and wavelength of the light. We used a refractive index of 1.55 for the precursor solution as determined for a 1.2 M solution with an Abbé refractometer (see Table S1). Since the spectrally averaged transflectance does not increase prior to the onset crystallization, we assume that the refractive index of the precursor solution remains mostly unchanged during the initial regimes of spin-coating (ejection and flow regimes). Using a broadbandight source, this will give rise to an interference pattern with a number of fringes/periods $(m)$ in a spectral interval $\left(\Delta v\right.$, in $\left.\mathrm{cm}^{-1}\right)$. The thickness $(d)$, can then be determined according to, ${ }^{53}$

$$
d=\frac{m}{2 \sqrt{\left(n^{2}+\sin ^{2} \theta\right)} \Delta v}
$$
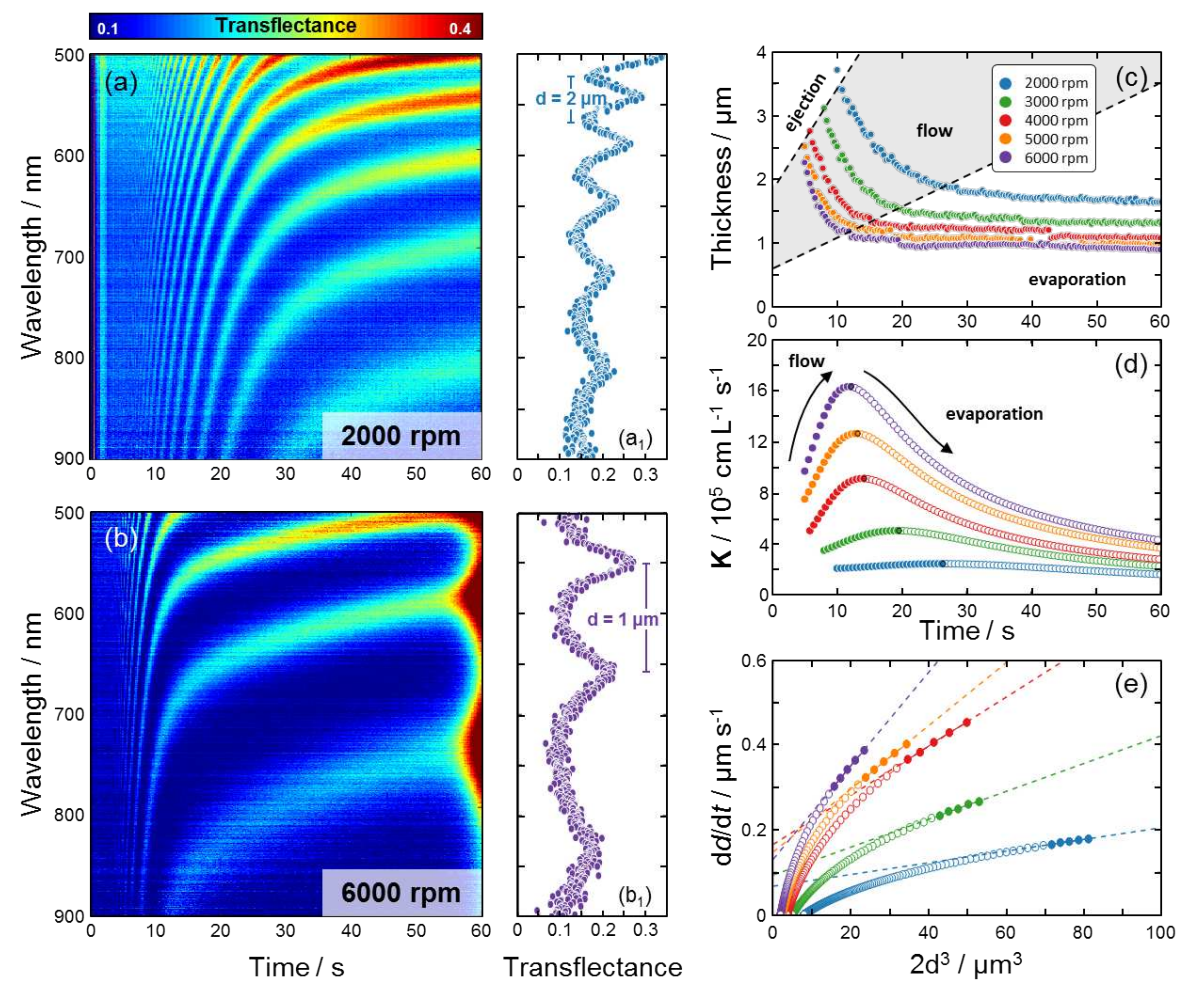

Figure 2. 2D Heat maps showing the evolution of the interference pattern with different spin-coating parameters of a $1.2 \mathrm{M}$ perovskite solution: (a) $2000 \mathrm{rpm}$ and (b) $6000 \mathrm{rpm}$. Both use a $5 \mathrm{~s}$ ramp. Both maps are plotted with the same $z$-scale according to the color bar at the top. $\left(a_{1}, b_{1}\right) 1 D$ spectra extracted at $t=18 \mathrm{~s}$ for both measurements showing the different number of fringes as well as the calculated thickness using Equation (2). (c) Evolution of absolute thickness for all spin-coating speeds with the different thinning regimes identified: ejection, flow, evaporation. (d) The flow constant, $\mathrm{K}$, extracted using Equation (4) plotted as a function of time where an increasing $\mathrm{K}$ indicates the regime when thinning is dominated 
The advantage of using a reflectance probe in the geometry shown in the inset in Figure $1 \mathrm{a}$ is that the incident angle $(\theta)$ is close to zero. This simplifies the calculation slightly by removing the necessity of estimating the angles of incidence and reflection in order to extract an accurate thickness. This leaves the refractive index ( $n$ ) the only variable introducing potential uncertainty in the determination of $d$. Measuring at normal incidence minimizes the thickness error due to an improper estimation of the refractive index since the light in this configuration travels the shortest path through the film that is characterized. We validated the absolute thickness extraction of the in-situ monitoring tool by measuring on a polymer film (Bisphenol A ethoxylate diacrylate) of known refractive index and thickness and establish an error of less than $2 \%$ (see $\mathrm{SI}$ ). Thus, under the condition that the refractive index is properly assessed, we can quite reliably extract the absolute thickness of a thin, semi-transparent film.

In Figure 2c, we plot the thickness evolution, extracted using Equation (2), during spin-coating at five spin-coating speeds ranging from 2000-6000 rpm. It is evident that a faster rotation speed not only leads to a quicker stabilization of the thickness but also results in thinner films in accordance with spin-coating theory. The thickness can only be extracted when at least one period (fringe) of the interference pattern is observable with several periods allowing for a more accurate thickness determination. This sets a thickness detection range based on how narrow fringes can be resolved (upper limit) and how broad a fringe can fit into the spectral range (lower limit). Another factor, which affects the initial thickness estimation, is how turbulent the surface is. Initially, solvent is ejected from the substrate, and it takes some time before thinning by flow initiates, which is when an interference pattern is first observed. We can therefore determine when thinning transitions from the ejection regime to the flow regime by timing the detection of a resolvable interference pattern. ${ }^{43,54}$

The ability to derive the wet film thickness $(d)$ or wet film thinning rate $\left(\frac{\boldsymbol{d} \boldsymbol{d}}{\boldsymbol{d} t}\right)$ from optical interferometry data enables the analysis of rheological parameters and transition from the flow regime to the evaporation regime. The model developed by Emslie, Bonner and Peck (EBP model) relates the wet-film thickness $(d)$ of a Newtonian liquid, which standard perovskite solutions have been shown to be, ${ }^{55}$ spin-cast at constant angular velocity $(\omega)$ to the solution density $(\rho)$ and viscosity $(\eta)$ according to, ${ }^{56}$

$d=\frac{d_{0}}{\left(1+4 K d_{0}^{2} t\right)^{1 / 2}}$

with the flow constant $K$ defined as:

$K=\rho \omega^{2} / 3 \eta$

Note that Equations ( 3 ) and (4) compare a relative change in the thickness, $d / d_{0}$, after a certain time, $t$, of spinning. In order to simplify this expression, we make the assumption that $4 K d_{0}^{2} t>1$, which removes $d_{0}$ from Equation (3) and enables to plot the dependence of the flow constant $K$ as a function of time (Figure $2 \mathrm{~d}$ ). For comparison, we determined density, viscosity, and refractive index of solutions with different precursor concentrations and the pure solvent mixture of DMF/DMSO by refractometry (Table 1). The results show that the refractive index and solution density vary linearly with concentration, while the dynamic viscosity depends on solution concentration in a more exponential fashion and is hence expected to dominate Equation (4) for higher concentrations also upon solvent evaporation during spin-coating.

Table 1. Table summarizing rheological properties of the precursor solutions at different concentrations. Refractive index was measured in an Abbé refractometer and the dynamic viscosity in a rolling ball viscometer

\begin{tabular}{|c|c|c|c|}
\hline c [mol/L] & $\begin{array}{c}\text { Refractive } \\
\text { index } n\end{array}$ & $\begin{array}{c}\text { Density } \boldsymbol{\rho} \\
{\left[\mathrm{g} / \mathrm{cm}^{3}\right]}\end{array}$ & $\begin{array}{c}\text { Dynamic } \\
\text { viscosity } \boldsymbol{\eta} \\
{[\mathrm{cP}]}\end{array}$ \\
\hline $\begin{array}{c}\text { DMF/DMSO } \\
4: 1 \mathrm{vol}\end{array}$ & $1.441 \pm 0.005$ & $0.96 \pm 0.02$ & 1.03 \\
\hline 0.2 & $1.458 \pm 0.005$ & $1.07 \pm 0.02$ & 1.37 \\
\hline 0.6 & $1.493 \pm 0.005$ & $1.24 \pm 0.03$ & $2.50 \pm 0.51$ \\
\hline 0.8 & $1.511 \pm 0.005$ & $1.31 \pm 0.03$ & $3.52 \pm 0.46$ \\
\hline 1 & $1.531 \pm 0.005$ & $1.45 \pm 0.01$ & $5.07 \pm 0.29$ \\
\hline 1.2 & $1.552 \pm 0.005$ & $1.53 \pm 0.02$ & $8.43 \pm 0.31$ \\
\hline
\end{tabular}

The initial solution viscosity determined from our results from the first measurement point of $K$ derived from optical interferometry (Figure 2d) and Equation (4) amount to $8.79 \pm 0.76 \mathrm{cP}$, which is in good agreement with the experimental viscosity of $8.43 \pm 0.31$ determined on solutions by rolling ball viscometry for the $1.2 \mathrm{M}$ solution summarized in Table 1. 
To include effects of solvent evaporation, Meyerhofer suggested a simplistic mathematical amendment of the EBP model by including solvent evaporation as a linear term, $E$, in the derivative form of Equation (3):

$\frac{d d}{d t}=-2 K d^{3}-E$

From plots of $\frac{d d}{d t}$, the flow constant $K$ and evaporation term $E$ can be estimated by linear extrapolation of the early stage of thinning as indicated in Figure 2e (all parameters in Table S2). The averaged values for the resulting viscosity determined from linear extrapolation of the initial stage of spin-coating from the Meyerhofer plots amount to $8.54 \pm 0.83 \mathrm{cP}$, which agrees well with the viscosity determined from rolling ball viscosimetry as well as evaluation of the flow constant (Figure $2 \mathrm{~d}$ ). The Meyerhofer plots show that the solution under investigation deviate strongly from linear behavior, which can be attributed to the complex composition of the precursor solutions containing a mixture of solvents (DMF and DMSO) as well as ionic precursors that can be expected to interact with solvents in different solution equilibria.

The time-dependency of $K$ shown in Figure $2 \mathrm{~d}$ also clearly illustrates the transition from the initial flow regime to the evaporation regime as the point where thinning by flow equals thinning by evaporation in the maximum of the curves. For the flow-regime, the solution concentration and hence density and refractive index can be assumed to not change considerably, indicating a dramatic change of the viscosity during hydrodynamic thinning of the wet film. We postulate that this is due to the gradient of rotational forces within the wet film from the layer adjacent to the rotating disk to the wet-film/air interface changes quite significantly when the wet-film layer is getting thinner during the flow-regime. ${ }^{57}$ In the evaporation dominated regime, the solution concentration will change, thus viscosity and density of the precursor solution increase. Our results, and especially the Meyerhofer plots, nicely illustrate differences in evaporation rate $E$ as function of the spin-coating speed. More elaborate analytical models that account for the physical phenomena in complex solutions such as perovskite precursor inks need to be developed in order to describe the full spin-coating process, which should include the more complex evaporation behavior of mixed solvent systems as well as concentration-dependence of density, viscosity and refractive index summarized in Table 1.

In summary, we here show how the thinning of the wet-film during spin-coating can be derived from optical interference measurements. Rheological parameters such as the solution viscosity can be reliably determined for the initial flow regime when solvent evaporation can be neglected. We illustrate that wet film thinning, and rheological parameters evolve in a complex manner, and more elaborate theory is required to relate experimental results to specific changes in solution rheology and composition upon solvent evaporation. This will likely require additional in-situ experiments that would also give insight into the early stages of the evolution of solution complexes into higher-order aggregated states.

\subsection{From thinning to crystallization: Effect of precursor concentration}

The onset of sample solidification (crystallization) is marked by less defined interference fringes, as shown in Figure 2 . This is due to the more absorbing and scattering perovskite thin-film, reducing the fraction of light reflected back to the detector significantly. This is where the power of our rather simple tool is demonstrated since we can, in the same measurement, monitor the thinning of the film, crystallization onset, and how the absorption further evolves with sub-second resolution. Note that in the detection geometry utilized, the absorption signal will be affected by changes in the absorptivity of the sample under investigation as well as changes in light scattering due to the formation of a rough surface upon crystallization. From the experimental data, the absolute amount of perovskite is difficult to determine, but relative changes in the evolving signal still provide significant insight into the formation kinetics.

In Figure 3, we compare the wet-film thinning and thin-film formation kinetics when spin-coating perovskite precursor solutions with varying concentration of $1.2 \mathrm{M}, 1.0 \mathrm{M}$ and $0.8 \mathrm{M}$. Figure $3 \mathrm{a}-\mathrm{c}$ shows the $2 \mathrm{D}$ plots of transflectance spectra acquired during spin-coating at $4000 \mathrm{rpm}$ as a function of spin-coating time (Figure 3a-c). Here, no anti-solvent drip was deposited during spin-coating as we aimed to investigate the unperturbed drying and crystallization process of the perovskite film. The initial wetfilm thinning is apparent in each 2D map as interference patterns. In Figure $3 d$, the variation in wet film thickness, $d$, calculated using Equation (2) with a time-invariant refractive index of 1.51, 1.53, and 1.55 as determined from refractometry reference experiments (see Table S1) is compared for the three different solution concentrations of $0.8 \mathrm{M}, 1.0 \mathrm{M}$ and $1.2 \mathrm{M}$, respectively. We observe that the rate of thinning increases with lower solution concentration, consistent with the lower solution viscosity, as determined by rolling ball viscometry summarized in Table S1. The wet-film thickness depends on solution concentration as expected. Optical monitoring provides direct feedback on the evolution of wet film thickness and allows the distinction between the ejection, flow, and evaporation regime of thin-film formation. This is important for e.g., timing and anti-solvent drop or transferring samples to a hotplate. In Figure $3 f$ and $g$ we show the top-view and cross-section SEM micrographs of the three films after annealing. Samples have a rough surface morphology, and the average height was determined to be $530 \mathrm{~nm}, 610 \mathrm{~nm}$, and $820 \mathrm{~nm}$ for the three precursor solutions in ascending order. The wet-film thickness of corresponding samples during spin-coating was estimated to be ca. $625 \mathrm{~nm}, 875 \mathrm{~nm}$, and $1235 \mathrm{~nm}$, which corresponds to a reduction in film thickness by $15 \%$ for the $0.8 \mathrm{M}$ solution and $34 \%$ for the higher solution concentration. We postulate that for higher solution concentrations, more solvent molecules remain bound by the perovskite precursor phase consistent with the lower wet film thinning rate and later onset of crystallization for the higher concentrated solution discussed below. 
The wet film thinning rate $\left(\frac{\boldsymbol{d} \boldsymbol{d}}{\boldsymbol{d} \boldsymbol{t}}\right)$ according to Equation (5) is shown as Meyerhofer plots for the three different precursor solution concentrations investigated, which is done by plotting the thinning rate as a function of the twice the thickness cubed $\left(2 d^{3}\right)$. The constants $K$ and $E$ from Equation (5) can then be determined from the slope of a linear fit (using the early data points) and the $y$-axis intercept, respectively. The viscosity is then determined from the slope to derive $K$ and Equation (4). By this procedure, the viscosities were determined to be $1.57 \mathrm{cP}$ for $0.8 \mathrm{M}, 2.94 \mathrm{cP}$ for $1.0 \mathrm{M}$, and $6.18 \mathrm{cP}$ for $1.2 \mathrm{M}$ solutions. Alternatively, the initial value of $K$ can be used, as was done in Figure 2, where the extracted viscosity amounts to $3.49 \mathrm{cP}, 4.83 \mathrm{cP}$, and $8.29 \mathrm{cP}$. These values all agree with the reference viscosity measurements (see Table 1), with a variation to an acceptable degree. As the viscosity may change during spin-coating, the slight discrepancy between the viscosities derived from first data points and Meyerhofer plots can be justified, but there can also be slight errors in the solution concentrations that would affect the result.
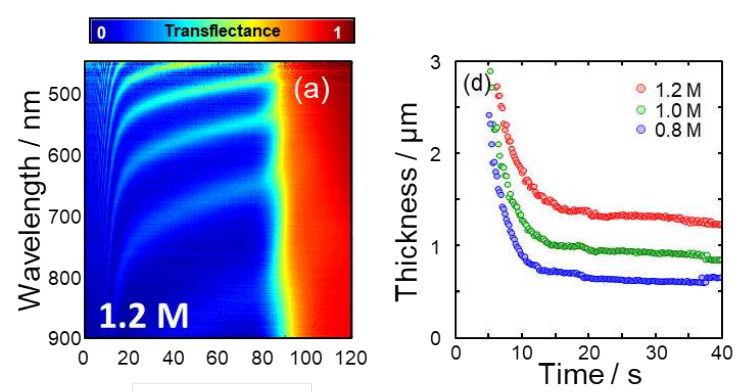

SEM images of thin films (post annealing)
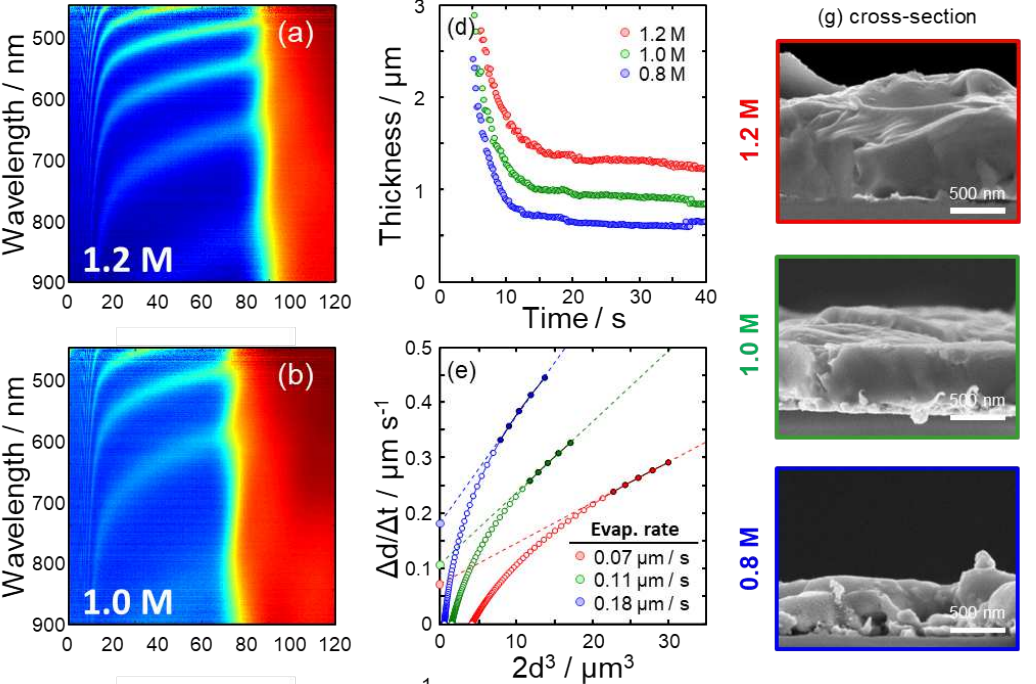

(f) top-view
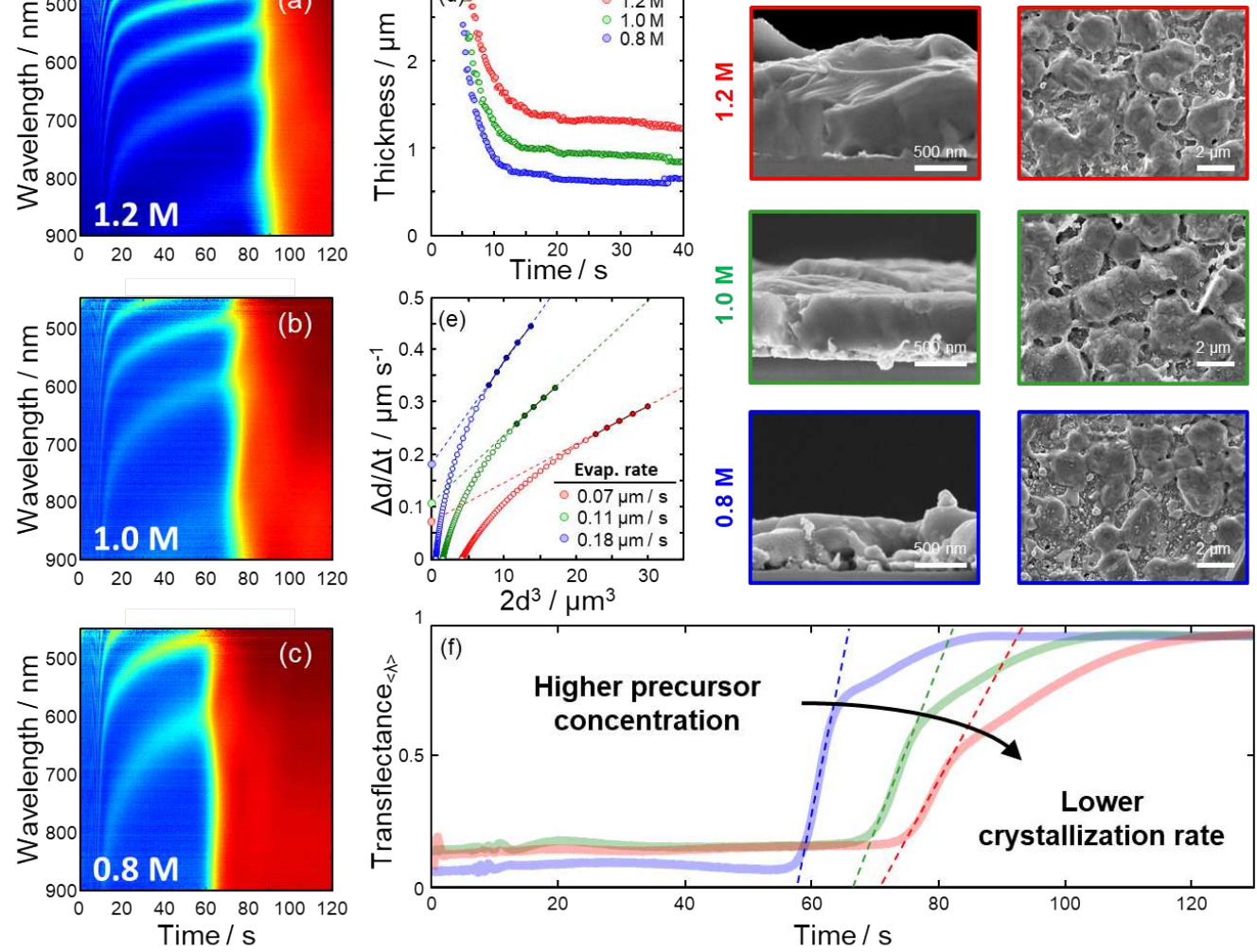

Figure 3. 2D transflectance heat maps during spin-coating for (a) $1.2 \mathrm{M}$, (b) $1.0 \mathrm{M}$, and (c) $0.8 \mathrm{M}$ precursor concentration. With decreasing concentration, the crystallization onset occurs earlier. (d) Extracted thickness prior to crystallization for the three concentrations. (e) Meyerhofer plots for the three different concentrations. The $y$-axis intercept of the linear extrapolation represents the evaporation rate. (f) Evolution of the spectrally averaged transflectance signal for the three measurements (colored lines). The dashed colored lines indicate that the rate of crystallization decreases with increasing concentration.

Figure $3 f$ displays the averaged transflectance signal to probe the onset of perovskite thin-film crystallization. Contrary to our initial intuition, that solutions with concentrations closer to supersaturation should exhibit an earlier onset of crystallization, precursor solutions with higher concentrations exhibit a later and less steep onset of crystallization also apparent from the 2D heat maps shown in Figure 3a-c. However, from the $y$-axis intercept of the linear extrapolation in the Meyerhofer representation of the thinning rate as a function of $d^{3}$, the evaporation constants $E$ was determined to be $0.07 \mu \mathrm{m} / \mathrm{s}$ for the $1.2 \mathrm{M}$ solution, $0.11 \mu \mathrm{m} / \mathrm{s}$ for the $1.0 \mathrm{M}$ solution and $0.18 \mu \mathrm{m} / \mathrm{s}$ for the $0.8 \mathrm{M}$ solution. From just the linear approximation of initial thinning behavior, the solution concentration can be expected to change more rapidly for the lower concentration solution in a given time interval. This indicates that solutions of lower concentration would reach oversaturation more rapidly, which is consistent with the earlier onset of crystallization. This is consistent with the considerably higher viscosity of higher concentration perovskite precursor solutions and also suggests a strong solvent-solute interaction in highly concentrated perovskite solutions with a substantial fraction of solvent molecules bound by perovskite precursor salts. ${ }^{29}$

From the changes in transflectance signals in Figure $3 \mathrm{f}$, the crystallization onset appears to proceed in two distinct stages and cannot be described by the Avrami model. ${ }^{58,59}$ In addition, the initial crystallization rate seems to depend on solution concentration. This suggests that the perovskite is formed faster from lower concentration precursor solutions, and more perovskite seems to be formed in the first stage of crystallization judging by the relative difference in signal intensity. As the 'triple cation' perovskite solution contains multiple species, we infer that crystallization proceeds via intermediates of different compositions, which will be further discussed in relation to the evolution of PL signals in the next section. 
We hypothesize that for lower concentration solutions, an excess solvent is more easily removed, and the precursor solution complexes are in an ideal state to form perovskite thin films. In addition, the evaporation of solvent molecules out of the wet-film from higher concentration solutions might be limited by mass transfer through the wet-film/air interface. ${ }^{60}$

The examples are shown here illustrate the capabilities of optical monitoring to track the evolution of thin-film formation with the potential to extract further details by more elaborate signal analysis, such as fitting the absorption signatures to evaluate the evolution of the bandgap and concentration and distinguishing scattering from absorption.

\subsection{Insight into anti-solvent induced crystallization with PL}

We investigated the effect of dispensing an anti-solvent drip during the spin-coating of the triple cation perovskite precursor solution. $1 \mathrm{~mL}$ of ethyl acetate was deposited at 35 seconds into the process. Figure 4a shows a 2D heat map of the evolving transflectance where, in contrast to data shown in Figure 2 and Figure 3, a clear absorption onset around $750 \mathrm{~nm}$ of the perovskite absorber can be observed after the anti-solvent drip is deposited. Note that these measurements were carried out with the reflective silver side of the substrate facing down. For this reason, the initial phase of spin-coating does not exhibit the interference patterns used for the determination of wet film thinning discussed in the previous two sections. In the supporting information, we are showing an example comparing the effect of the silver mirror on the substrate facing up or down.

The 2D heat map suggests the presence of periodic signatures that may stem from interference fringes indicating that the sample has a smooth surface morphology after anti-solvent drip deposition, which is corroborated by a low transflectance signal below the bandgap.

In Figure $4 \mathrm{~b}$ we show a 2D heat map of the PL evolution of the perovskite during spin coating on a glass substrate where an anti-solvent drip is applied at 35 seconds to induce crystallization. The expected perovskite PL signature of the material composition investigated would be a peak around $760 \mathrm{~nm}$. Immediately after the anti-solvent drip, PL emission appears at shorter wavelengths (around $720 \mathrm{~nm}$ ), after which it slowly red-shifts throughout the $180 \mathrm{~s}$ spin-coating period (peak position is indicated in the PL heat map with a black dashed line). It is noteworthy that even after nearly 3 minutes of spin-coating, the peak has not reached the expected position at $760 \mathrm{~nm}$ and is still red-shifting. Since $180 \mathrm{~s}$ is far longer than typical spin-coating recipes, ${ }^{61}$ it suggests the formation of the perovskites is ongoing long after an anti-solvent drip has been applied and first completed during annealing, as discussed in the next section.

We calculated the relative PL quantum yield (PLQY) by dividing the transflectance signal measured at the excitation wavelength $(470 \mathrm{~nm}$ ) with the integrated PL signal. Figure 4c shows the evolution of this relative PLQY after the AS-drip has been applied where two distinct peaks with similar relative PLQY at around $45 \mathrm{~s}$ and $90 \mathrm{~s}$ are observed. Both the transflectance and photoluminescence spectra show that the bandgap of the first and second rel. PLQY maxima change towards lower bandgaps (red and green traces in Figures $4 \mathrm{e} \& 4 \mathrm{f}$ ). There is also a noticeable difference with respect to the spectra acquired at the end of spincoating at $180 \mathrm{~s}$ (blue trace, Figures $4 \mathrm{e} \& 4 \mathrm{f}$ ).

The evolution of absorption onset was evaluated from the inflection point of the absorption onset (acquired by taking the derivative of the transflectance spectrum) as proposed by Kirchartz et al. ${ }^{62}$ The evolution of the absorption onset correlates with the evolution of the PL peak position extracted from fitting an asymmetric Voigt line shape to the spectrum (Figure $4 \mathrm{~d}$ ). As the thin film crystallizes, the difference between absorption onset and PL peak position the two diminishes. Comparing Figures $4 \mathrm{e} \& 4 \mathrm{f}$, we can see that the absorption onset gradually shifts toward lower energies, whereby re-absorption of the PL could be an explanation for why the PL spectrum at $180 \mathrm{~s}$ (blue) compared to that at $90 \mathrm{~s}$ (green) has a lower intensity at shorter wavelengths.

It is generally assumed that the anti-solvent induces almost instantaneous crystallization within a few seconds..$^{33,63}$ Our results unravel an indeed quite complex formation mechanism. The rapid increase of PL intensity indicates a rather instantaneous crystallization of perovskite crystals on the surface of the wet-film induced by the anti-solvent drip. ${ }^{64,65}$ These initial crystals exhibit a high PLQY and higher bandgap indicating the formation of bromide-rich perovskite seed crystals. We interpret the intermittent decrease in rel. PLQY as the partial dissolution of the initial nuclei in the remaining solvent. ${ }^{21,66-68}$ Consecutively, more iodide becomes incorporated into the perovskite phase indicated by the spectral redshift of the second PL intensity maximum shown in Figure 4. The consecutive decrease in PL intensity is consistent with the formation of a continuous perovskite thin film. Charge carriers are increasingly likely to recombine non-radiatively as they can reach defects in adjacent grains via diffusion. ${ }^{69,70}$ We summarize these processes in the schematic in $4 \mathrm{~g}$. Our result highlight the value of optical monitoring to track the evolution of compositional changes during film formation.

Note that in standard spin-coating procedures, the sample would be moved to a hotplate for thermal annealing after $40 \mathrm{~s}$ of spin-coating. This would likely lead to the thermal quenching of samples in a state where not all iodide is incorporated into the perovskite phase, which we will illuminate further in follow up work to this study. Hirselandt et al. (see submitted draft) discusses the role and effect of anti-solvent drip timing on the film formation mechanism and resulting device performance variation comparing the "triple cation" recipe investigated here also to compositionally simpler methylammonium lead iodide ( $\mathrm{MAPbl}_{3}$ ) precursor solutions. [Hirselandt et al., see manuscript included for referees] 

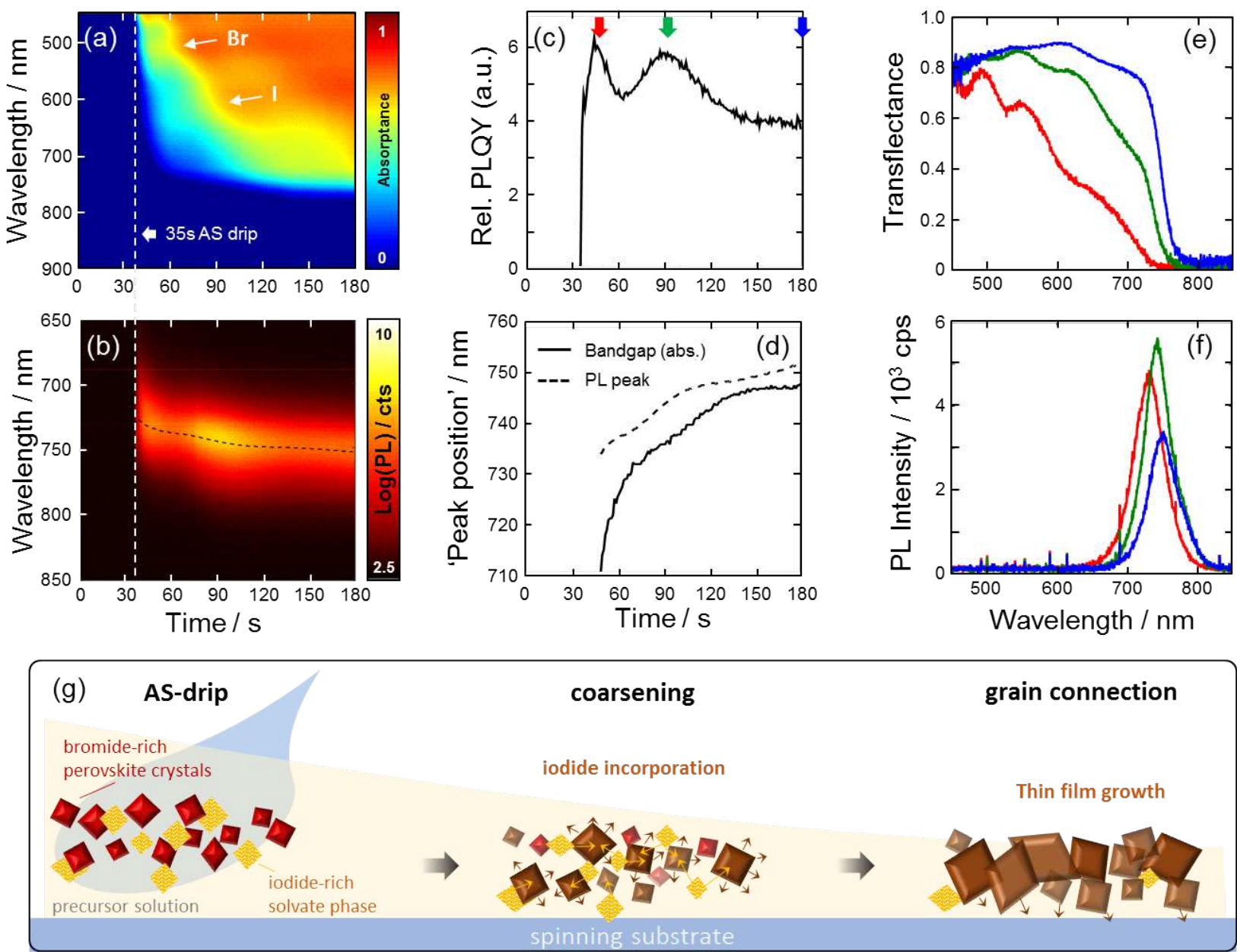

Figure 4. 2D heat maps of (a) transflectance and (b) PL extracted during spin-coating of the perovskite on glass for $180 \mathrm{~s}$ where an anti-solvent (ethyl acetate) was dripped at $35 \mathrm{~s}$ to induce crystallization. (c) PL peak intensity (red circles) and peak asymmetry coefficient (blue circles) for the entire measurement extracted using the asymmetric Voigt line shape (Equations (6) and (7)). (d) Comparison between the PL peak position extracted from peak fitting (dashed line) and the absorption onset (solid line) is representative of the PL peak. (e) Transflectance and (f) PL spectra at the three points in the time indicated by the colored arrows in panel (c). (g) Schematic showing the formation process during spin-coating as the precursor solution converts into a crystallized film prior to annealing.

\subsection{Describing the full process}

As a concluding step, we here summarize the insight gained from the analysis of optical features recorded during spin-coating discussed in the previous section and also include the annealing step for the standard "tripe-cation perovskite" recipe widely employed in the literature. ${ }^{18,61}$ This recipe is commonly used for the fabrication of both $p$-i- $n$ and $n$-i-p perovskite solar cells, enabling power conversion efficiencies $>20 \%$ (see $\mathrm{SI}$ ). ${ }^{11}$ Ethyl acetate is used as anti-solvent which is applied after $25 \mathrm{~s}$ of spincoating at $4000 \mathrm{rpm}$ ( $5 \mathrm{~s} \mathrm{ramp}$ ) After AS treatment, spin-coating continues for an additional 15 seconds before the sample is transferred to the hotplate for an annealing step at $100^{\circ} \mathrm{C}$ for 1 hour (transfer taking a few seconds). The middle and bottom rows show the transflectance and $\mathrm{PL}$ data respectively for both spin-coating and annealing.

The thickness of the wet film is around $1.25 \mu \mathrm{m}$ prior to the deposition of the anti-solvent (Figure 5a). After, the interference pattern changes, although it remains rather constant for the remaining $15 \mathrm{~s}$ of spin-coating, suggesting a drastic change in the refractive index and/or thickness of the film. As discussed in the previous section, the PL peak position evolves from $720 \mathrm{~nm}$ right after the deposition of the anti-solvent drip (Figure $5 \mathrm{c}$ ). Compared to a later drip time of $35 \mathrm{~s}$ used in the previous section, the peak width stabilizes quite quickly, where there is still some asymmetry observed.

When transferring the sample to the hotplate, the absorption signal rapidly becomes dominated by spectral features that indicate fast and complete crystallization of a perovskite thin film within 5 seconds of annealing. It is evident that the main transformation to a perovskite thin film occurs within the first few seconds of annealing, which can also be observed by the naked eye. In comparison, the PL peak (Figure $5 \mathrm{~d}$ ) first broadens and then becomes narrower during annealing. We note that the PL intensity during spin-coating was substantially higher than during annealing, and results shown utilized exposure times 4 times higher in order to detect a measurable signal. The PL intensity exhibits a drastic reduction of the PL yield in correlation with the crystallization of the film measured with UV-Vis (Figure $5 b$ ). 
The increasing absorptance measured below the bandgap (> $760 \mathrm{~nm}$ ) can be explained by the emerging microstructure of the film, which causes the incoming light to scatter more. ${ }^{71}$ The reduced PL yield can be an effect of charge carriers more effectively diffusing to defects in a fully condensed thin film ${ }^{46,72,73}$ but could also reflect changes in the escape probability of photons out of the crystalline thin film. In addition, this could be due to change in the doping density of thin films during annealing as discussed by Suchan et al. While we here opted for a fixed annealing time, follow up work correlating spectral changes during annealing with resulting solar cell devices performance could be very interesting to further optimize the preparation protocol.

Spin-coating
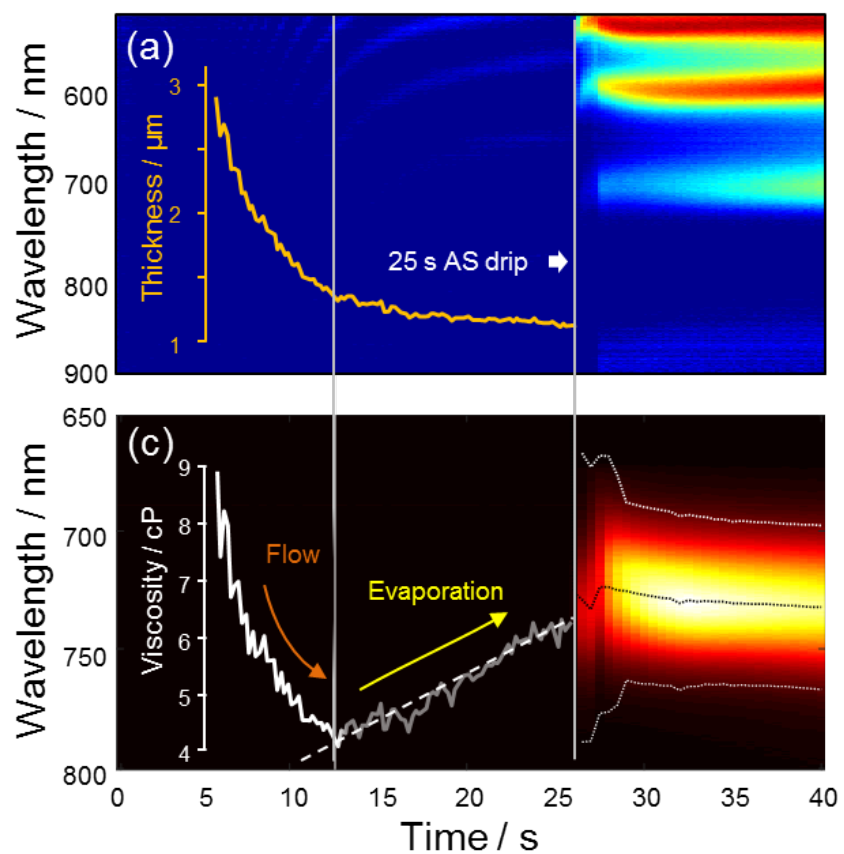

Annealing
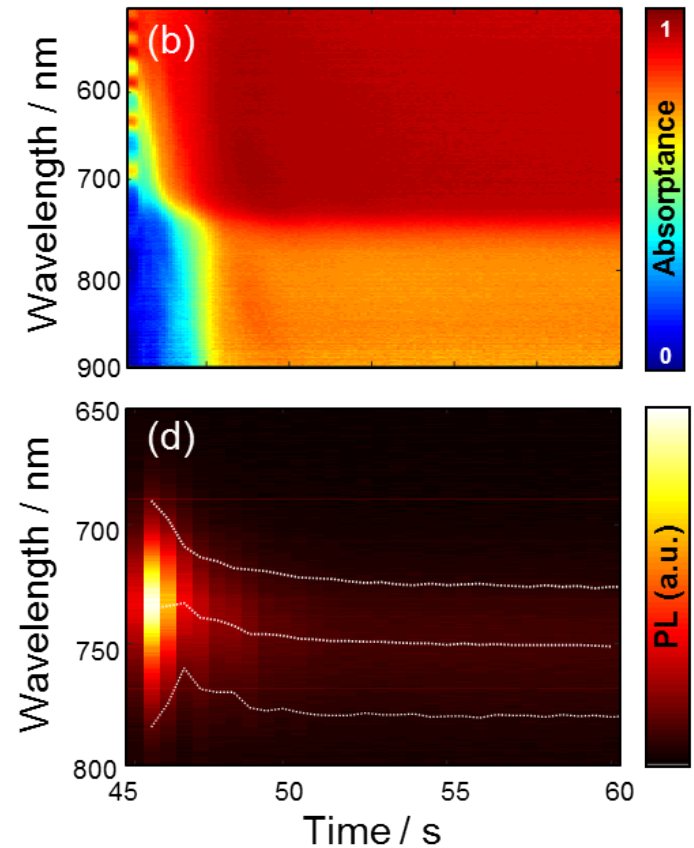

Figure 5. Transflectance during (a) spin-coating and (b) annealing. The thickness is extracted from the interference fringes prior to the AS drip, which is only faintly observed in (a). $2 \mathrm{D}$ heat maps showing the evolution of PL during (c) spin-coating and (d) annealing. The calculated viscosity is shown in (c) , where we indicate the transition between thinning being dominated by flow to evaporation (orange and yellow lines). 2D heat maps showing the PL evolution during (c) spin-coating and (d) annealing where the dotted lines relate to the PL peak and its widths acquired from fitting an asymmetric Voigt line shape. 


\section{Conclusions}

We demonstrate a facile way to in-situ process monitoring for the optimization of deposition strategies and gaining a deeper understanding of formation mechanisms. Our fibre-optics based setup was designed to have a small footprint to be easy to integrate into common process equipment such as spin-coaters and slot-die coaters. Using this setup, we here present our insight gained on key formation stages from solution to thin film of "triple cation" perovskite semiconductors. We demonstrate a novel approach to acquiring the dynamic viscosity optically, which provides an easy way to assess rheological properties of precursor inks under relevant process conditions. Counterintuitively, we show that the crystallization onset occurs later for precursor solutions with higher concentration, which we attribute to a strong interaction of ionic precursors with solvent molecules and mass transfer limitation of solvents out of the wet film during spin-coating. With photoluminescence (PL), we were able to demonstrate the formation of intermediate species and how these contribute to the relative PL yield. Such measurements may be of importance in optimizing the open-circuit voltage as it directly relates to the PL quantum yield. Being able to control these properties at an early stage of device processing is of crucial importance. We foresee that optical monitoring tools like the one presented here will become increasingly used for studying processes as well as quality control in industrial manufacturing. With such a small footprint and ease of implementation, this tool can be readily integrated into many experimental setups to give insight into different stages of solution processing of semiconductors. We are currently conducting several follow-up projects in our team e.g., on the formation of mixed bromide/iodide semiconductors from solution ${ }^{74}$, the timing of the anti-solvent drip on film formation and device performance (Hirselandt et al., see paper attached), the effect of interrupting spincoating at different points in time on device performance, and we are integrating the setup into our slot-die coater for in-line process monitoring.

\section{Conflicts of interest}

There are no conflicts to declare.

\section{Acknowledgements}

A.M. and K.H. thank Johannes Sutter for providing samples for thickness validation. A.M., K.H., J.D., R.M., F.M., and E.U. acknowledge funding from the German Ministry of Education and Research (BMBF) for the Young Investigator Group Hybrid Materials Formation and Scaling (HyPerFORME) within the program "NanoMatFutur" (grant no. 03XP0091). J.Z.L. acknowledges funding from the Chinese Scholarship Council (CSC, grant No. CSC201908120116). E.U. acknowledges funding from the Swedish Research Council (Projects 2015-00163 and 2018-05014).

\section{Notes and references}

Y. Liu, Y. Zhang, Z. Yang, H. Ye, J. Feng, Z. Xu, X. Zhang, R. Munir, J. Liu, P. Zuo, Q. Li, M. Hu, L. Meng, K. Wang, D.-M. Smilgies, G. Zhao, H. Xu, Z. Yang, A. Amassian, J. Li, K. Zhao and S. Liu, Nat. Commun., 2018, 9, 5302. L. Xu, S. Yuan, H. Zeng and J. Song, Mater. Today Nano, 2019, 6, 100036.

3 M. A. Green, A. Ho-Baillie and H. J. Snaith, Nat. Photonics, 2014, 8, 506.

4 Best Resarch-Cell Efficiency Chart | Photovoltaic Research | NREL.

5 J. Dagar, K. Hirselandt, A. Merdasa, A. Czudek, R. Munir, F. Zu, N. Koch, T. Dittrich and E. L. Unger, Sol. RRL, 2019, 3, 1900088.

6 J. Li, R. Munir, Y. Fan, T. Niu, Y. Liu, Y. Zhong, Z. Yang, Y. Tian, B. Liu, J. Sun, D.-M. Smilgies, S. Thoroddsen, A. Amassian, K. Zhao and S. F. Liu, Joule, , DOI:10.1016/j.joule.2018.04.011.

7 S. Das, B. Yang, G. Gu, P. C. Joshi, I. N. Ivanov, C. M. Rouleau, T. Aytug, D. B. Geohegan and K. Xiao, ACS Photonics, 2015, 2, 680-686.

8 R. Patidar, D. Burkitt, K. Hooper, D. Richards and T. Watson, Mater. Today Commun., 2020, 22, 100808.

$9 \quad$ F. Mathies, E. J. W. List-kratochvil and E. L. Unger, Energy Technol., 2019, 1900991.

10 M. Saliba, T. Matsui, J.-Y. Seo, K. Domanski, J.-P. Correa-Baena, N. Mohammad K., S. M. Zakeeruddin, W. Tress, A. Abate, A. Hagfeldt and M. Grätzel, Energy Environ. Sci., , DOI:10.1039/C5EE03874J.

11 M. Saliba, J. P. Correa-Baena, C. M. Wolff, M. Stolterfoht, N. Phung, S. Albrecht, D. Neher and A. Abate, Chem. Mater., 2018, 30, 4193-4201.

12 N. J. Jeon, J. H. Noh, Y. C. Kim, W. S. Yang, S. Ryu and S. II Seok, Nat. Mater., 2014, 13, 897-903.

13 M. Xiao, F. Huang, W. Huang, Y. Dkhissi, Y. Zhu, J. Etheridge, A. Gray-Weale, U. Bach, Y.-B. Cheng and L. Spiccia, Angew. Chemie, 2014, 126, 10056-10061. J. Li, R. Yang, L. Que, Y. Wang, F. Wang, J. Wu and S. Li, J. Mater. Res., 2019, 34, 2416-2424. 
F. Yang, G. Kapil, P. Zhang, Z. Hu, M. A. Kamarudin, T. Ma and S. Hayase, ACS Appl. Mater. Interfaces, 2018, 10, 16482-16489.

Z. Zhang, X. Luo, J. Ding and J. Zhang, J. Solid State Chem., 2019, 274, 199-206.

D. Prochowicz, M. M. Tavakoli, A. Solanki, T. W. Goh, K. Pandey, T. C. Sum, M. Saliba and P. Yadav, J. Mater. Chem. A, 2018, 6, 14307-14314.

M. Saliba, T. Matsui, J. Y. Seo, K. Domanski, J. P. Correa-Baena, M. K. Nazeeruddin, S. M. Zakeeruddin, W. Tress, A. Abate, A. Hagfeldt and M. Grätzel, Energy Environ. Sci., 2016, 9, 1989-1997.

F. Wang, T. Zhang, Y. Wang, D. Liu, P. Zhang and H. Chen, J. Mater. Chem. A Mater. energy Sustain., 2019, 7, 12166-12175.

K. Bruening and C. J. Tassone, J. Mater. Chem. A, 2018, 6, 18865-18870.

T. Song, Z. Yuan, F. Babbe, D. P. Nenon, E. Aydin, S. De Wolf and C. M. Sutter-fella, , DOI:10.1021/acsaem.9b02052. X. Zheng, B. Chen, C. Wu and S. Priya, Nano Energy, 2015, 17, 269-278.

M. Jung, S.-G. Ji, G. Kim and S. II Seok, Chem. Soc. Rev, 2019, 48, 2011-2038.

W. A. Dunlap-Shohl, Y. Zhou, N. P. Padture and D. B. Mitzi, Chem. Rev., 2019, 119, 3193-3295.

M. Jung, S. G. Ji, G. Kim and S. II Seok, Chem. Soc. Rev., 2019, 48, 2011-2038.

S. Rahimnejad, A. Kovalenko, S. M. Forés, C. Aranda and A. Guerrero, ChemPhysChem, 2016, 2795-2798.

E. Radicchi, E. Mosconi, F. Elisei, F. Nunzi and F. De Angelis, ACS Appl. Energy Mater., , DOI:10.1021/acsaem.9b00206.

Y. Xia, C. Ran, Y. Chen, Q. Li, N. Jiang, C. Li, Y. Pan, T. Li, J. P. Wang and W. Huang, J. Mater. Chem. A, , DOI:10.1039/c6ta09554b.

M.-C. Tang, D. Barrit, R. Munir, R. Li, J. M. Barbé, D.-M. Smilgies, S. Del Gobbo, T. D. Anthopoulos and A. Amassian, Sol. RRL, 2019, 3, 1800305.

D. Barrit, A. D. Sheikh, R. Munir, J. M. Barbé, R. Li, D.-M. Smilgies and A. Amassian, J. Mater. Res., , DOI:10.1557/jmr.2017.117.

S. Masi, A. Rizzo, R. Munir, A. Listorti, A. Giuri, C. Esposito Corcione, N. D. Treat, G. Gigli, A. Amassian, N. Stingelin and S. Colella, Adv. Energy Mater., , DOI:10.1002/aenm.201602600.

X. Zhang, R. Munir, Z. Xu, Y. Liu, H. Tsai, W. Nie, J. Li, T. Niu, D. Smilgies, M. G. Kanatzidis, A. D. Mohite, K. Zhao, A. Amassian and S. F. Liu, Adv. Mater., , DOI:10.1002/adma.201707166.

R. Munir, A. D. Sheikh, M. Abdelsamie, H. Hu, L. Yu, K. Zhao, T. Kim, O. El Tall, R. Li, D. M. Smilgies and A. Amassian, Adv. Mater., 2017, 29, 1604113.

P. P. Khlyabich and Y.-L. Loo, Chem. Mater., 2016, 28, 9041-9048.

F. Babbe and C. M. Sutter-fella, 2020, 1903587, 1-17.

K. Suchan, J. Just, P. Becker, E. L. Unger and T. Unold, J. Mater. Chem. A, 2020, 25-30.

B. Tripathi, S. Huettner and F. Panzer, , DOI:10.1039/c9ta12409h.

M.-C. Tang, D. Barrit, R. Munir, R. Li, J. M. Barbé, D.-M. Smilgies, S. Del Gobbo, T. D. Anthopoulos and A. Amassian, Sol. RRL, 2019, 3, 1800305.

M. Qin, K. Tse, T.-K. Lau, Y. Li, C.-J. Su, G. Yang, J. Chen, J. Zhu, U.-S. Jeng, G. Li, H. Chen and X. Lu, Adv. Mater., 2019, 31, 1901284.

A. Z. Chen, B. J. Foley, J. H. Ma, M. R. Alpert, J. S. Niezgoda and J. J. Choi, J. Mater. Chem. A, 2017, 5, 7796-7800. O. Filonik, M. E. Thordardottir, J. Lebert, S. Pröller, S. Weiß, L. J. Haur, A. Priyadarshi, P. Fontaine, P. MüllerBuschbaum, N. Mathews and E. M. Herzig, Energy Technol., 2019, 7, 1900343.

Y. Zhong, R. Munir, J. Li, M.-C. Tang, M. R. Niazi, D.-M. Smilgies, K. Zhao and A. Amassian, ACS Energy Lett., 2018, 3, 1078-1085.

R. Munir, A. D. Sheikh, M. Abdelsamie, H. Hu, L. Yu, K. Zhao, T. Kim, O. E. Tall, R. Li, D.-M. Smilgies and A. Amassian, Adv. Mater., , DOI:10.1002/adma.201604113.

M. Chauhan, Y. Zhong, K. Schötz, B. Tripathi, A. Köhler, S. Huettner and F. Panzer, J. Mater. Chem. A, , DOI:10.1039/c9ta12409h.

E. L. Unger, A. R. Bowring, C. J. Tassone, V. L. Pool, A. Gold-Parker, R. Cheacharoen, K. H. Stone, E. T. Hoke, M. F. Toney and M. D. McGehee, Chem. Mater., 2014, 26, 7158-7165.

H. Cao, J. Li, Z. Dong, J. Su, J. Chang, Q. Zhao, Z. Li, L. Yang and S. Yin, ACS Energy Lett., 2019, 4, 2821-2829.

W.-A. Quitsch, D. W. DeQuilettes, O. Pfingsten, A. Schmitz, S. Ognjanovic, S. Jariwala, S. Koch, M. Winterer, D. S. Ginger and G. Bacher, J. Phys. Chem. Lett., 2018, 9, 2062-2069.

A. Merdasa, A. Kiligaridis, C. Rehermann, M. Abdi-Jalebi, J. Stöber, B. Louis, M. Gerhard, S. D. Stranks, E. L. Unger and I. G. Scheblykin, ACS Energy Lett., 2019, 4, 1370-1378.

S. Chen, X. Wen, S. Huang, F. Huang, Y. Cheng, M. Green and A. Ho-Baillie, Sol. RRL, 2016, 1-8.

R. S. Sanchez and E. Mas-Marza, Sol. Energy Mater. Sol. Cells, 2016, 158, 189-194.

A. Merdasa, M. Bag, Y. Tian, E. Källman, A. Dobrovolsky and I. G. Scheblykin, J. Phys. Chem. C, 2016, 120, 1071110719.

M. Buchhorn, S. Wedler and F. Panzer, J. Phys. Chem. A, 2018, 122, 9115-9122. 
N. J. Harrick, Appl. Opt., 1971, 10, 2344.

M. Abdelsamie, K. Zhao, M. R. Niazi, K. W. Chou and A. Amassian, J. Mater. Chem. C, $2014,2,3373-3381$.

A. Giuri, E. Saleh, A. Listorti, S. Colella, A. Rizzo, C. Tuck and C. E. Corcione, , DOI:10.3390/nano9040582.

A. G. Emslie, F. T. Bonner and L. G. Peck, J. Appl. Phys., 1958, 29, 858-862.

K. Wei Chou, H. Ullah Khan, M. R. Niazi, B. Yan, R. Li, M. M. Payne, J. E. Anthony, D.-M. Smilgies and A. Amassian, J. Mater. Chem. C, 2014, 2, 5681-5689.

Q. Hu, L. Zhao, J. Wu, K. Gao, D. Luo, Y. Jiang, Z. Zhang, C. Zhu, E. Schaible, A. Hexemer, C. Wang, Y. Liu, W. Zhang, M. Grätzel, F. Liu, T. P. Russell, R. Zhu and Q. Gong, Nat. Commun., , DOI:10.1038/ncomms15688.

S. Ahmad, P. K. Kanaujia, W. Niu, J. J. Baumberg and G. Vijaya Prakash, ACS Appl. Mater. Interfaces, 2014, 6, 10238-10247.

C. J. Lawrence, , DOI:10.1063/1.866986.

M. Saliba, J. P. Correa-Baena, C. M. Wolff, M. Stolterfoht, N. Phung, S. Albrecht, D. Neher and A. Abate, Chem. Mater., 2018, 30, 4193-4201.

L. Krückemeier, U. Rau, M. Stolterfoht and T. Kirchartz, , DOI:10.1002/aenm.201902573.

K. Wang, M.-C. Tang, H. X. Dang, R. Munir, D. Barrit, M. De Bastiani, E. Aydin, D.-M. Smilgies, S. De Wolf and A. Amassian, Adv. Mater., 2019, 31, 1808357.

A. Z. Chen, M. Shiu, X. Deng, M. Mahmoud, D. Zhang, B. J. Foley, S.-H. Lee, G. Giri and J. J. Choi, Chem. Mater., 2019, 31, 1336-1343.

B. J. Foley, S. Cuthriell, S. Yazdi, A. Z. Chen, S. M. Guthrie, X. Deng, G. Giri, S.-H. Lee, K. Xiao, B. Doughty, Y.-Z. Ma and J. J. Choi, Nano Lett., 2018, 18, 6271-6278.

T. Udayabhaskararao, M. Kazes, L. Houben, H. Lin and D. Oron, Chem. Mater., 2017, 29, $1302-1308$.

H. Gao, C. Bao, F. Li, T. Yu, J. Yang, W. Zhu, X. Zhou, G. Fu and Z. Zou, ACS Appl. Mater. Interfaces, 2015, 7, 91109117.

N. T. K. Thanh, N. Maclean and S. Mahiddine, Chem. Rev., 2014, 114, 7610-7630.

P. Fassl, Y. Zakharko, L. M. Falk, K. P. Goetz, F. Paulus, A. D. Taylor, J. Zaumseil and Y. Vaynzof, J. Mater. Chem. C, 2019, 7, 5285-5292.

S. G. Motti, D. Meggiolaro, S. Martani, R. Sorrentino, A. J. Barker, F. De Angelis and A. Petrozza, Adv. Mater., 2019, 31, 1901183.

Y. Tian and I. G. Scheblykin, J. Phys. Chem. Lett., 2015, 6, 3466-3470.

H. Khassaf, S. K. Yadavalli, Y. Zhou, N. P. Padture and A. I. Kingon, J. Phys. Chem. C, 2019, $123,24$.

A. Sharenko and M. F. Toney, J. Am. Chem. Soc., 2016, 138, 463-470.

C. Rehermann, A. Merdasa, K. Suchan and E. L. Unger, submitted, 2020, 1-32. 


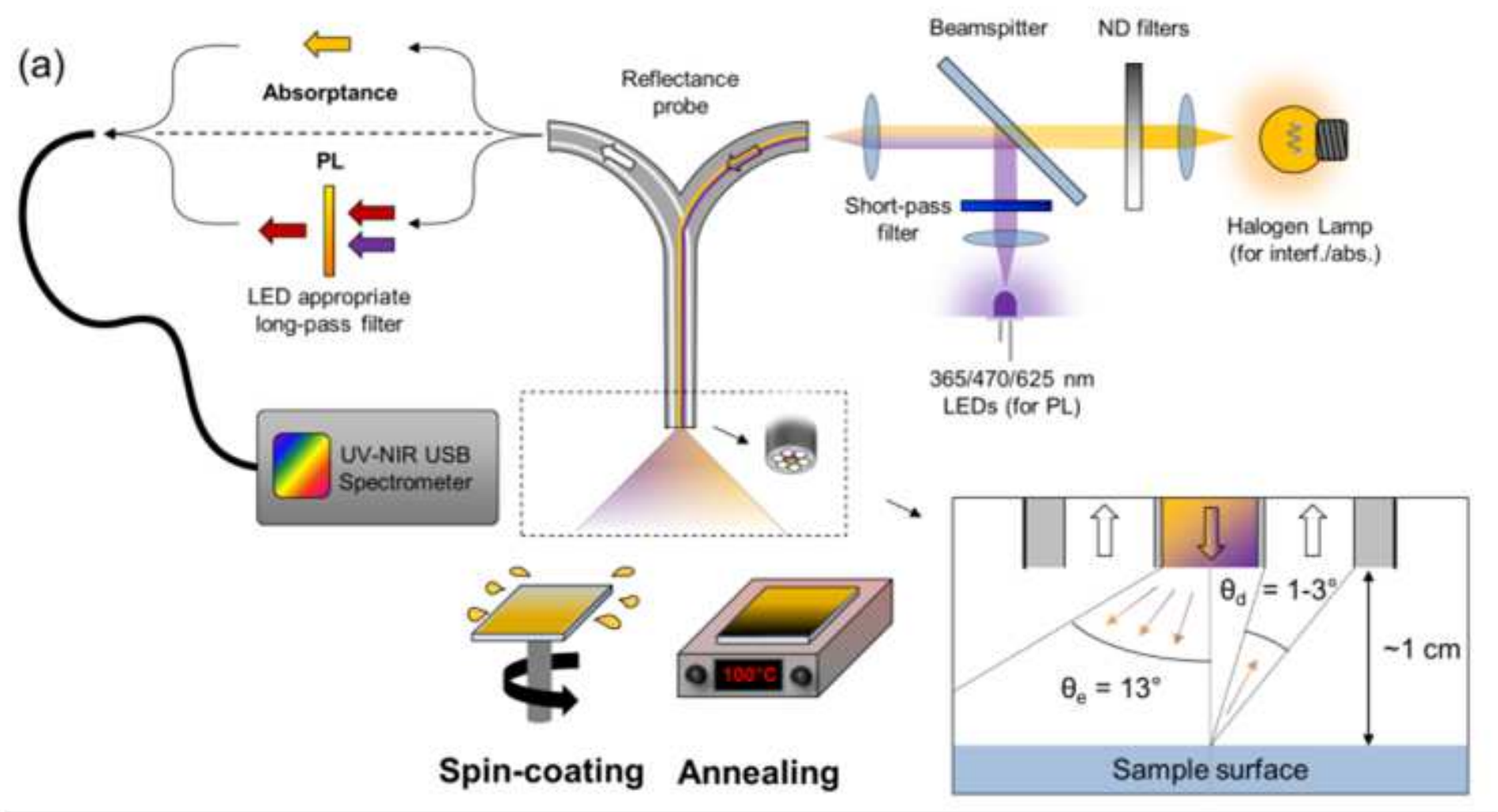

(b) Interference

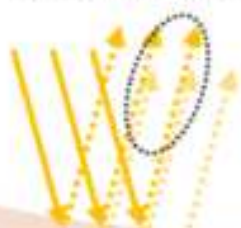

Absorptance

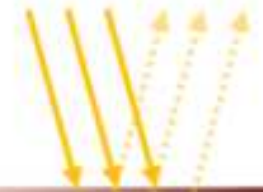

Solution

Photoluminescence

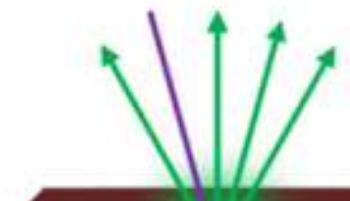

Film

\section{Figure 1}

(a) Schematic representation of the measurement setup in which only the reflectance probe is inside the glovebox. A white light source (halogen lamp) is used for absorptance measurements a,nd one of three available LEDs (emission peak at $365 \mathrm{~nm}, 470 \mathrm{~nm}$, or $625 \mathrm{~nm}$ ) is used for photoluminescence (PL) generation. The reflectance probe can be placed directly above either the spin-coater or hot plate. Light is detected with a USB spectrometer (Ocean Insight) prior to which an LED appropriate long-pass filter is placed for PL measurements to filter out the excitation. (b) Schematic demonstrating the thinning and progression from solution to a crystallized film during spin-coating. The schematic also illustrates the three types of measurements we can employ during this process, where we can measure the interference pattern (extracting thickness), the absorptance, or in a separate measurement, the PL. 

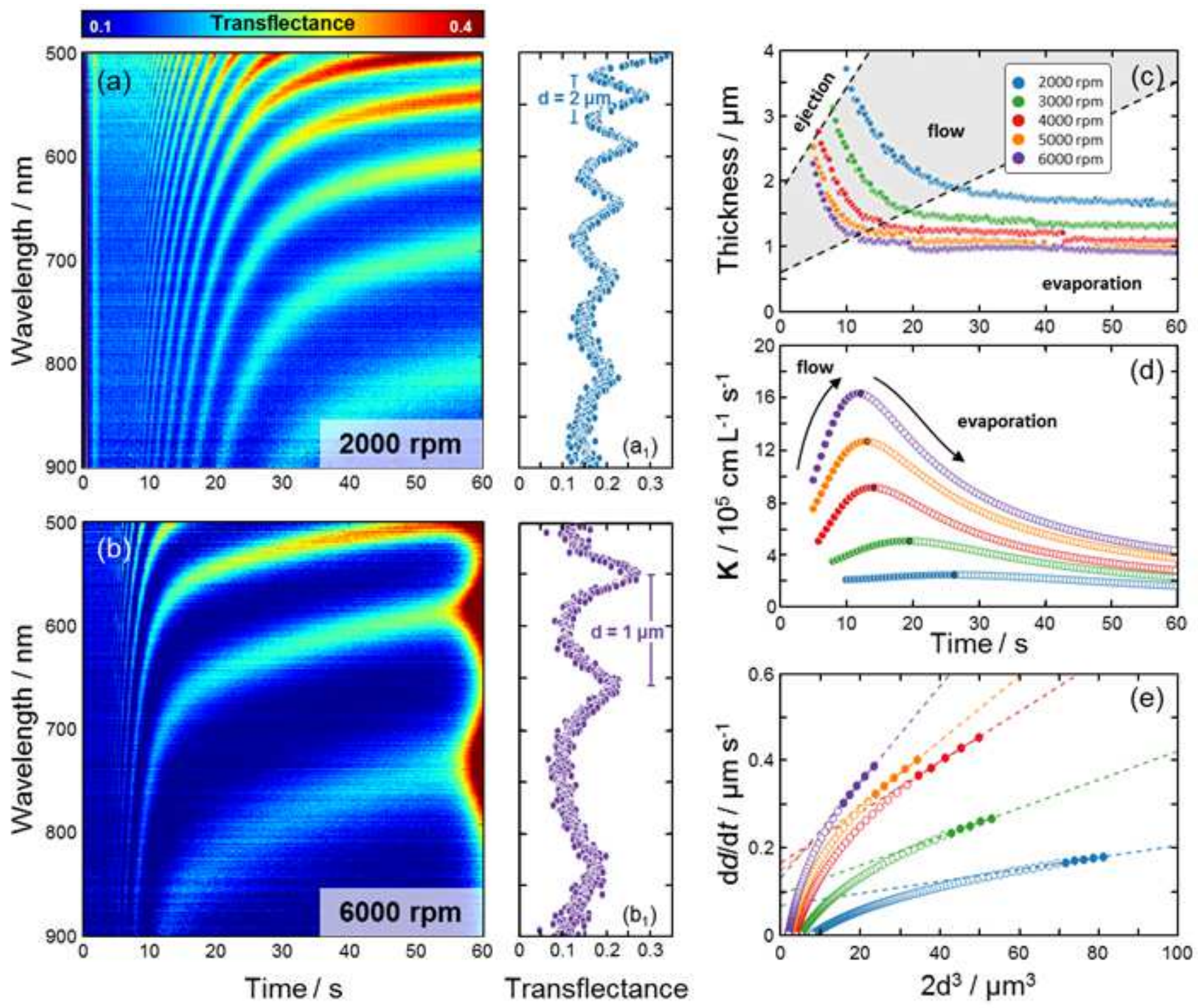

Figure 2

2D Heat maps showing the evolution of the interference pattern with different spin-coating parameters of a $1.2 \mathrm{M}$ perovskite solution: (a) $2000 \mathrm{rpm}$ and (b) $6000 \mathrm{rpm}$. Both use a $5 \mathrm{~s}$ ramp. Both maps are plotted with the same $z$-scale according to the color bar at the top. (a1,b1) 1D spectra extracted at $t=18 \mathrm{~s}$ for both measurements showing the different number of fringes as well as the calculated thickness using Equation (2). (c) Evolution of absolute thickness for all spin-coating speeds with the different thinning regimes identified: ejection, flow, evaporation. (d) The flow constant, K, extracted using Equation (4) plotted as a function of time where an increasing $\mathrm{K}$ indicates the regime when thinning is dominated by flow (solid circles) and each measurement where linear fits are made to the first five data points (indicated by solid circles). The slope of the line determines the flow constant, $\mathrm{K}$, from which viscosity can be extracted. 


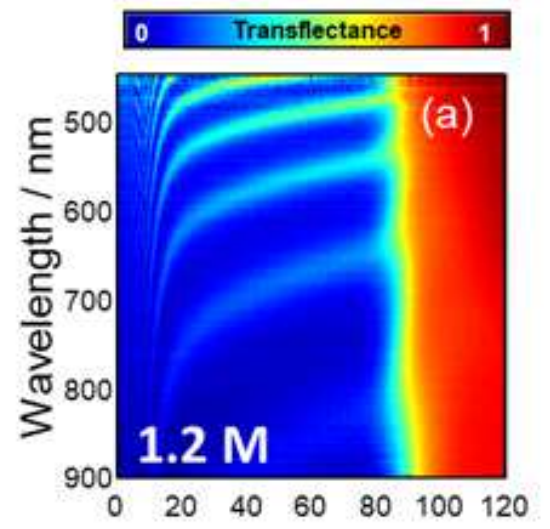

SEM images of thin films (post annealing)

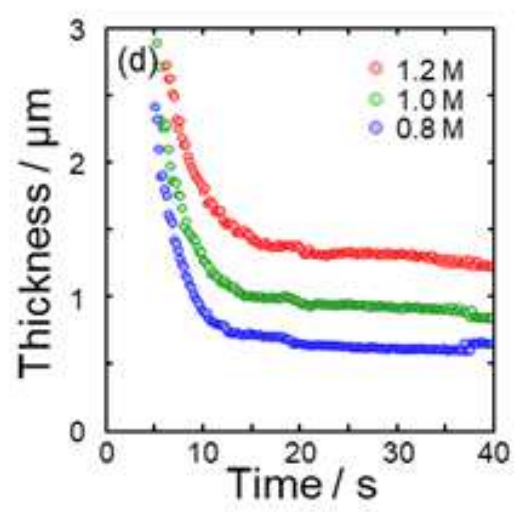

(g) cross-section
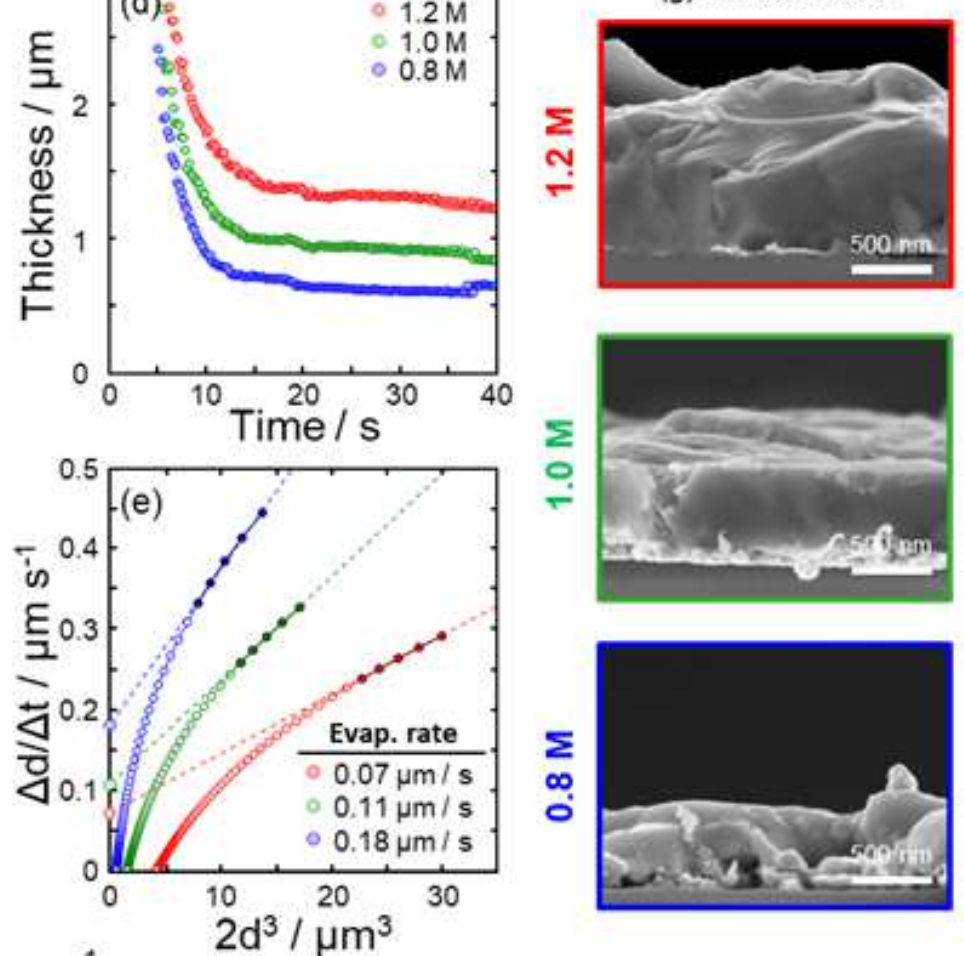

(f) top-view
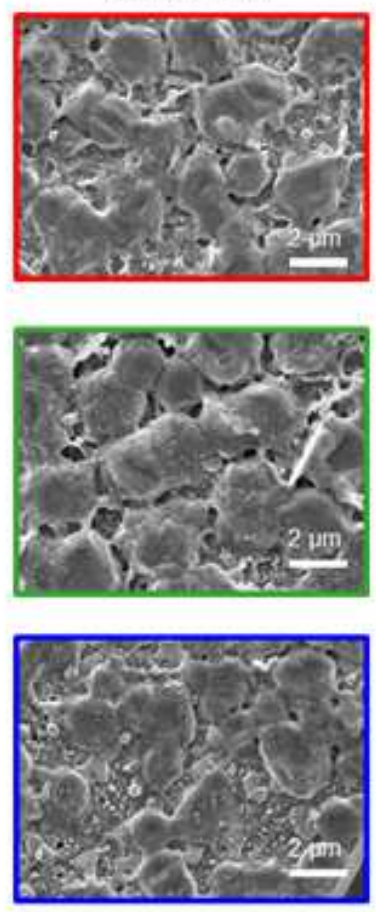
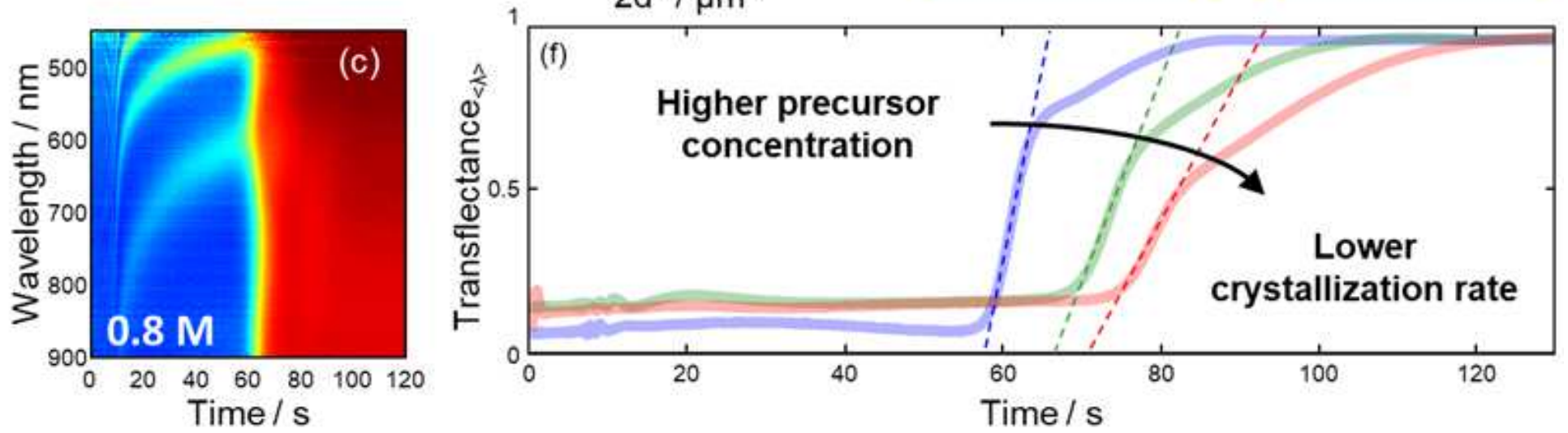

Figure 3

2D transflectance heat maps during spin-coating for (a) $1.2 \mathrm{M}$, (b) $1.0 \mathrm{M}$, and (c) $0.8 \mathrm{M}$ precursor concentration. With decreasing concentration, the crystallization onset occurs earlier. (d) Extracted thickness prior to crystallization for the three concentrations. (e) Meyerhofer plots for the three different concentrations. The y-axis intercept of the linear extrapolation represents the evaporation rate. (f) Evolution of the spectrally averaged transflectance signal for the three measurements (colored lines). The dashed colored lines indicate that the rate of crystallization decreases with increasing concentration. 

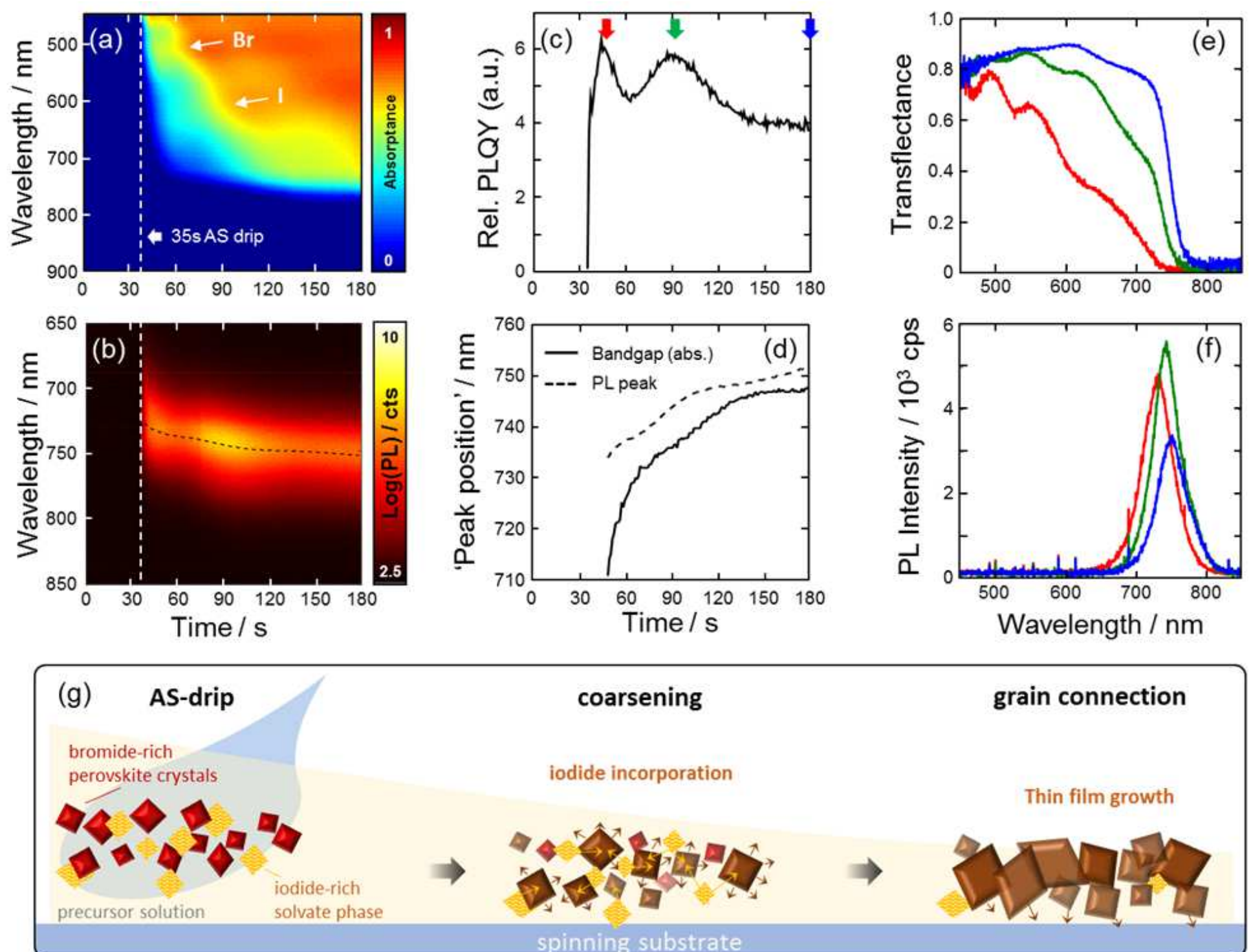

\section{Figure 4}

2D heat maps of (a) transflectance and (b) PL extracted during spin-coating of the perovskite on glass for $180 \mathrm{~s}$ where an anti-solvent (ethyl acetate) was dripped at $35 \mathrm{~s}$ to induce crystallization. (c) PL peak intensity (red circles) and peak asymmetry coefficient (blue circles) for the entire measurement extracted using the asymmetric Voigt line shape (Equations (6) and (7)). (d) Comparison between the PL peak position extracted from peak fitting (dashed line) and the absorption onset (solid line) is representative of the PL peak. (e) Transflectance and (f) PL spectra at the three points in the time indicated by the colored arrows in panel (c). (g) Schematic showing the formation process during spin-coating as the precursor solution converts into a crystallized film prior to annealing. 


\section{Spin-coating}

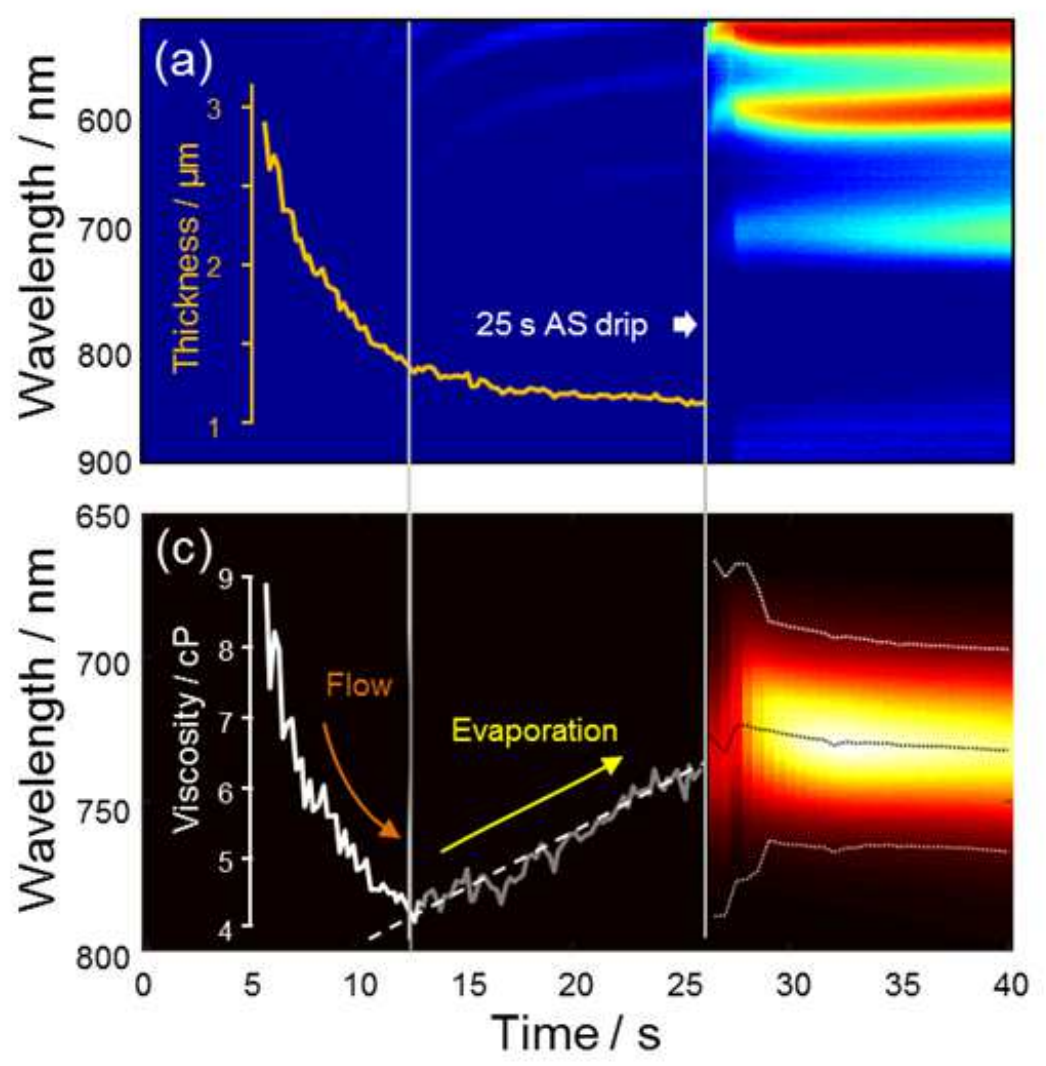

\section{Annealing}
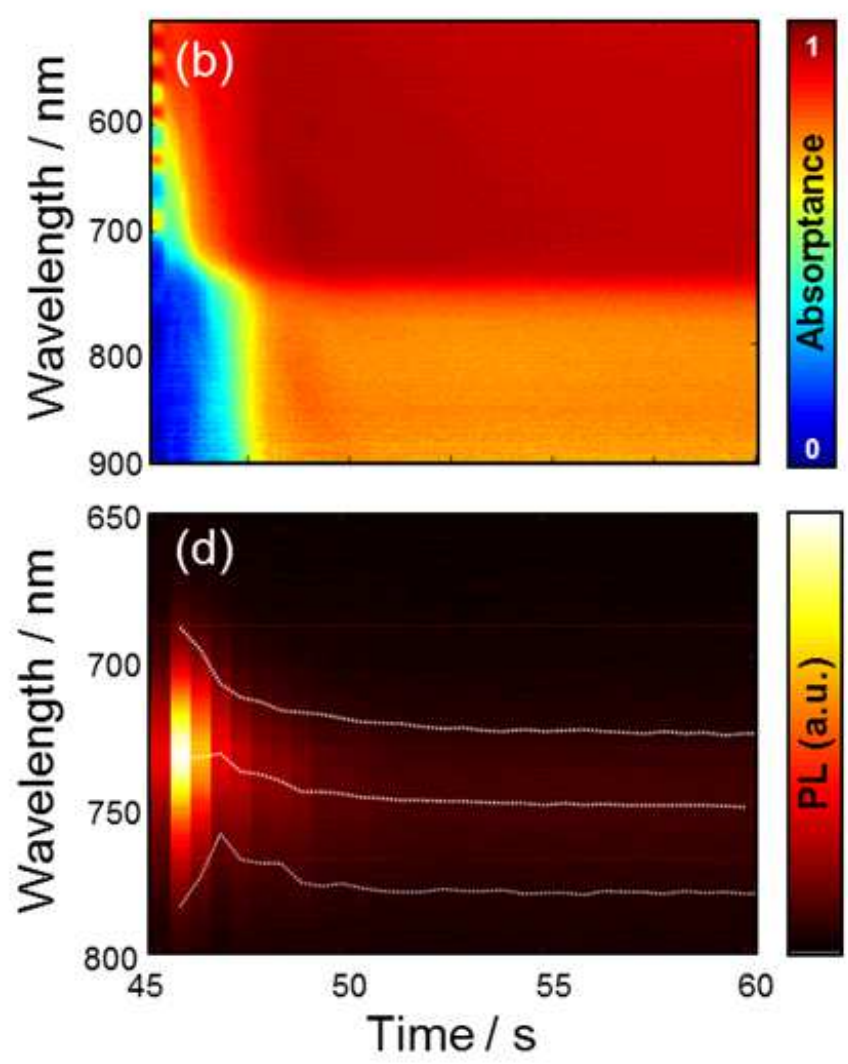

\section{Figure 5}

Transflectance during (a) spin-coating and (b) annealing. The thickness is extracted from the interference fringes prior to the AS drip, which is only faintly observed in (a). 2D heat maps showing the evolution of PL during (c) spin-coating and (d) annealing. The calculated viscosity is shown in (c) ,where we indicate the transition between thinning being dominated by flow to evaporation (orange and yellow lines). 2D heat maps showing the PL evolution during (c) spin-coating and (d) annealing where the dotted lines relate to the PL peak and its widths acquired from fitting an asymmetric Voigt line shape.

\section{Supplementary Files}

This is a list of supplementary files associated with this preprint. Click to download.

- Merdasa3CATformationSI.pdf 